\title{
Fundamentals of reservoir surface energy as related to surface properties, wettability, capillary action, and oil recovery from fractured reservoirs by spontaneous imbibition
}

\author{
DE-FC26-03NT15408 \\ Semi-Annual Report \\ 01/01/2006 - 06/30/2006 \\ Norman R. Morrow, Principal Investigator \\ Herbert Fischer \\ Yu Li \\ Geoffrey Mason \\ Douglas Ruth \\ Siddhartha Seth \\ Jason Zhengxin Tong \\ Peigui Yin \\ Shaochang Wo
}

August 2006

Re-submitted September 2006

Submitted by:

Chemical \& Petroleum Engineering

University of Wyoming

Dept. 3295, 1000 E. University Ave

Laramie, WY 82071 


\section{Disclaimer}

This report was prepared as an account of work sponsored by an agency of the United States Government. Neither the United States Government nor any agency thereof, nor any of their employees, makes any warranty, express or implied, or assumes any legal liability or responsibility for the accuracy, completeness, or usefulness of any information, apparatus, product, or process disclosed, or represents that its use would not infringe privately owned rights. Reference herein to any specific commercial product, process, or service by trade name, trademark, manufacturer, or otherwise does not necessarily constitute or imply its endorsement, recommendation, or favoring by the United States Government or any agency thereof. The views and opinions of authors expressed herein do not necessarily state or reflect those of the United States Government or any agency thereof. 


\begin{abstract}
The objective of this project is to increase oil recovery from fractured reservoirs through improved fundamental understanding of the process of spontaneous imbibition by which oil is displaced from the rock matrix into the fractures. Spontaneous imbibition is fundamentally dependent on the reservoir surface free energy but this has never been investigated for rocks. In this project, the surface free energy of rocks will be determined by using liquids that can be solidified within the rock pore space at selected saturations. Thin sections of the rock then provide a two-dimensional view of the rock minerals and the occupant phases. Saturations and oil/rock, water/rock, and oil/water surface areas will be determined by advanced petrographic analysis and the surface free energy which drives spontaneous imbibition will be determined as a function of increase in wetting phase saturation. The inherent loss in surface free energy resulting from capillary instabilities at the microscopic (pore level) scale will be distinguished from the decrease in surface free energy that drives spontaneous imbibition.
\end{abstract}

A mathematical network/numerical model will be developed and tested against experimental results of recovery versus time over broad variation of key factors such as rock properties, fluid phase viscosities, sample size, shape and boundary conditions. Two fundamentally important, but not previously considered, parameters of spontaneous imbibition, the capillary pressure acting to oppose production of oil at the outflow face and the pressure in the non-wetting phase at the no-flow boundary versus time, will also be measured and modeled. Simulation and network models will also be tested against special case solutions provided by analytic models.

In the second stage of the project, application of the fundamental concepts developed in the first stage of the project will be demonstrated. The fundamental ideas, measurements, and analytic/numerical modeling will be applied to mixed-wet rocks. Imbibition measurements will include novel sensitive pressure measurements designed to elucidate the basic mechanisms that determine induction time and drive the very slow rate of spontaneous imbibition commonly observed for mixed-wet rocks. In further demonstration of concepts, three approaches to improved oil recovery from fractured reservoirs will be tested; use of surfactants to promote imbibition in oil wet rocks by wettability alteration: manipulation of injection brine composition: reduction of the capillary back pressure which opposes production of oil at the fracture face. 


\section{TABLE OF CONTENTS}

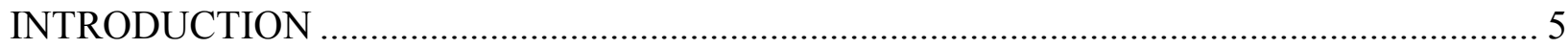

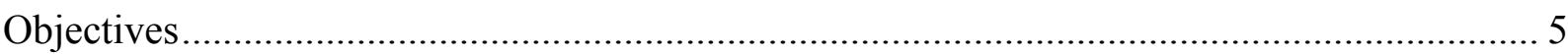

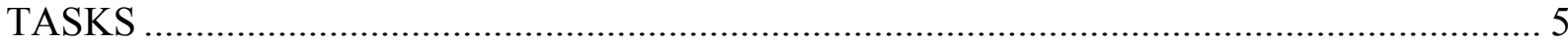

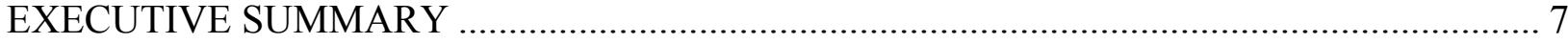

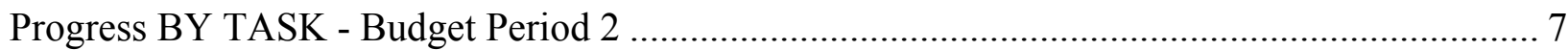

Task 6. Rock preparation and Work of displacement and surface areas ................................ 7

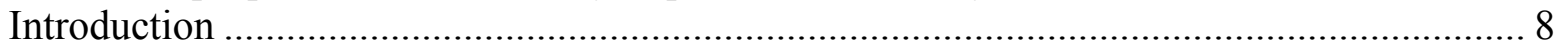

Experimental Procedures: Capillary Pressure and Interfacial Areas..................................... 9

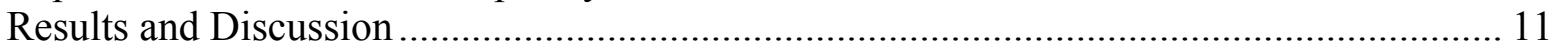

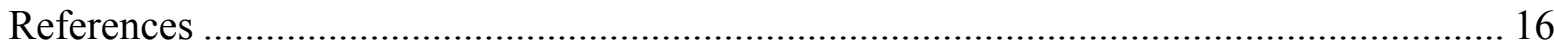

Task 7. Novel imbibition measurements on mixed-wet rock and network models.................... 24

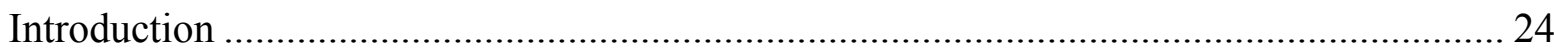

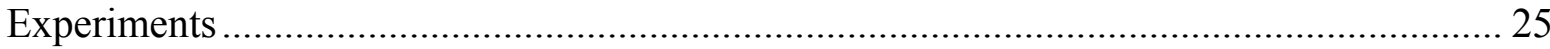

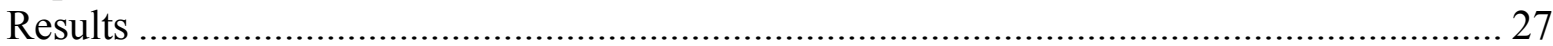

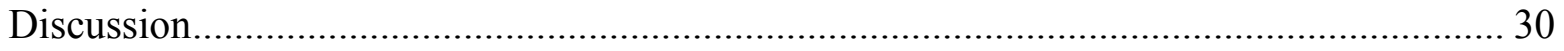

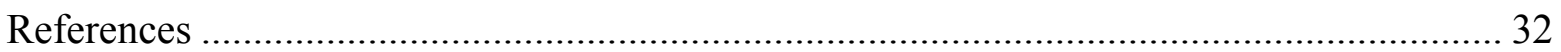

Task 8. Application of network/numerical model to mixed wet rocks. .................................... 38

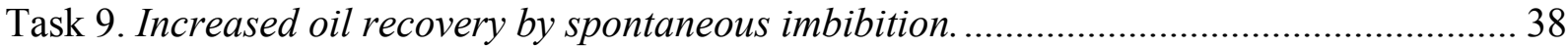

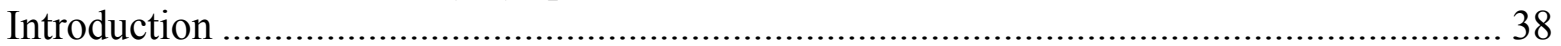

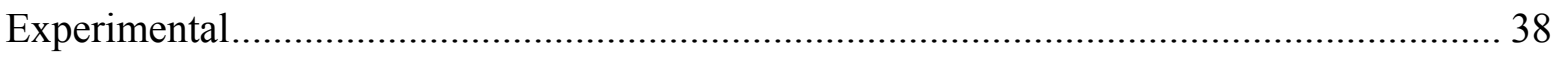

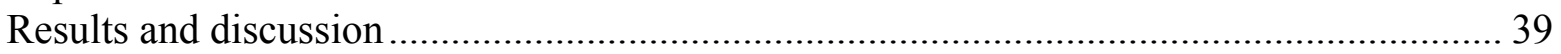

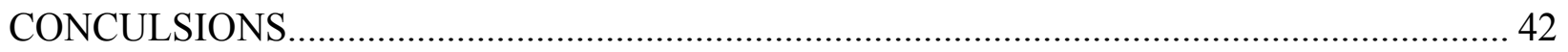




\section{INTRODUCTION}

\section{Objectives}

The long-range objective of this project is to improve oil recovery from fractured reservoirs through improved fundamental understanding of the process of spontaneous imbibition by which oil is displaced from the rock matrix into the fractures. Spontaneous imbibition is fundamentally dependent on the surface energy. An initial objective is to determine the surface energy and relate the dissipation of surface energy to the mechanism of spontaneous imbibition. A parallel objective is to model the mechanism of spontaneous imbibition by a combination of network analysis and numerical modeling. Also fundamentally important, but not previously considered, parameters of spontaneous imbibition, the capillary pressure acting to oppose production of oil at the outflow face and the pressure in the non-wetting phase at the no-flow boundary (in effect within oil in the non-invaded zone of the rock matrix) versus time, will also be measured and compared with values predicted by the mathematical model. The next objective is to measure surface energy and related spontaneous imbibition phenomena for mixed-wettability rocks prepared by adsorption from crude oil. The dissipation of surface free energy must then be related to oil production at mixed-wet conditions. The final objective is to apply the results of the project to improved oil recovery from fractured reservoirs in three ways: reduction of the capillary force that opposes oil production at the fracture face; change in wettability towards increased water wetness; identification of conditions where choice of invading brine composition can give improved recovery.

\section{TASKS}

\section{Budget period 1, July 1, 2003 through June 30, 2005 - Ideas and Concept development: Fundamentals of Spontaneous Imbibition}

Task 1. Work of displacement and surface free energy. Obtain complementary sets of capillary pressure drainage and imbibition data and data on changes in rock/brine, rock/oil, and oil/brine interfacial areas with change in saturation for drainage and imbibition for at least two rock types (sandstone and carbonate). Determine free-energy/work-of-displacement efficiency parameters for drainage and imbibition for at least two rock types so that changes in rock/wetting phase/nonwetting phase surface areas can be closely estimated from capillary pressure measurements.

Task 2. Imbibition in simple laboratory and mathematical network models. Study imbibition in at least three simple tube networks that can be modeled analytically to establish and/or confirm fundamental aspects of the pore scale mechanism of dynamic spontaneous imbibition with special emphasis on determining how spontaneous imbibition is initiated and the key factors in how the saturation profile develops with time. Incorporate rules developed from laboratory measurements on relatively simple networks into the design of a computational network model. Use the network model to obtain an account of the mechanism by which imbibition is initiated, the saturation profile is developed, and the rate of spontaneous imbibition in terms of the dissipation of surface free energy that accompanies change in saturation.

Task 3. Novel observations on fluid pressures during imbibition and the mechanism of nonwetting phase production at the imbibition face. Make novel observations on the imbibition 
mechanism including details of the mechanism of oil production at the outflow rock face and the change in the non-wetting phase pressure at the no-flow boundary of the core during the course of spontaneous imbibition for at least 16 distinct combinations of rock/ fluid properties.

Task 4. Network/numerical model and new imbibition data. Develop a numerical simulator specifically designed for spontaneous imbibition. Incorporate the network model to obtain a network/numerical model that includes matching the measured pressure in the non-wetting phase at the no-flow boundary, and the pressure that opposes production of oil at the open rock face. Imbibition data will be obtained for at least 10 rocks with over six-fold variation in permeability, and at least 6 orders of magnitude variation in viscosity ratio, and at least 10 variations in sample size, shape, and boundary conditions.

Task 5. Comparison with similarity solutions. Compare results given by simulation with special case analytic results given by similarity solutions for spontaneous imbibition for at least five distinct cases of rock and fluid properties.

\section{Budget Period 2, July 1, 2005 through June 30, 2008 - Demonstration of concept: Application to mixed wettability rocks and improved oil recovery from fractures reservoirs.}

Task 6. Rock preparation and Work of displacement and surface areas

Obtain a range of rock types and identify and obtain crude oils that induce stable mixed wettability. Prepare at least 25 rocks with mixed wettability through crude oil/brine/rock interactions. Determine work of displacement for drainage and imbibition and measure the variation in rock/brine, rock/oil, and oil/brine interfacial areas during the course of drainage and imbibition for at least two examples of mixed wettability.

Task 7. Novel imbibition measurements on mixed-wet rock and network models. Obtain, for at least six mixed-wet rocks, spontaneous imbibition data that includes measurements of the nonwetting phase pressure at the no-flow boundary, observations on the capillary pressure that resists production at the open rock face.

Task 8. Application of network/numerical model to mixed wet rocks. Use network models to relate dissipation of surface energy to rate of spontaneous imbibition and to account for the frequently observed induction time prior to the onset of spontaneous imbibition into mixed wettability rocks.

Task 9. Increased oil recovery by spontaneous imbibition. The mechanism of increased recovery from mixed wet rocks by use of surfactants that promote spontaneous imbibition by favorable wettability alteration will be investigated for at least four distinct examples of crude $\mathrm{oil} / \mathrm{brine} / \mathrm{rock} / \mathrm{surfactant}$ combinations. The mechanism of increased recovery by manipulation of brine composition will be investigated for at least four crude oil/brine/rock combinations. Addition of very low concentration surfactants to the imbibing aqueous phase will be explored as a means of increasing the rate of oil recovery by reducing the capillary forces which resist production of oil at the fracture face. At least twelve combinations of rock and fluid properties including both very strongly wetted and mixed wet rocks will be tested. 


\title{
EXECUTIVE SUMMARY
}

The first section of this report contains a complete account of results on relationships between work of displacement and the inherent loss of free energy by spontaneous imbibition. Data reported for both sandstones and carbonates. The efficiency of conversion of work to surface energy is lowest for carbonates and is ascribed to the high aspect ratio of pores formed by dissolution of fossils.

A new method of measuring the effective capillary pressure at the imbibition front is reported. Results are included for production of both essentially incompressible (oil) and compressible (air) non-wetting phases. The effective capillary pressures at the front are essentially constant with respect to rate of advance of the front. A network/numerical model of imbibition data for mixed wet rocks is currently being tested against recently obtained data for a range of initial conditions.

Studies on promotion of spontaneous imbibition are reported in which the presence of divalent ions on the effectiveness of surfactants were tested. Results obtained to date show that a trend of increase in recovery recently reported for chalk was not observed for the limestone. Measurements of relationships between oil recovery by spontaneous imbibition and brine composition are in progress for mixed-wet rocks that show strong response of waterflood recovery to change in salinity.

\section{PROGRESS BY TASK - BUDGET PERIOD 2}

\section{Task 6. Rock preparation and Work of displacement and surface areas}

\begin{abstract}
The increase in surface energy resulting from drainage of a wetting phase from a porous medium is often equated to the work of displacement determined from the area under its capillary pressure curve. However, capillary pressure versus saturation relationships are not reversible and do not represent quasistatic displacement. The increase in surface energy is less than the work done because of inherent capillary instabilities that are the basic cause of capillary pressure hysteresis. Nevertheless, identification of the area under a capillary pressure curve with thermodynamic work of displacement can be justified by interpreting the curve as a series of alternating isons (reversible displacements) and rheons (spontaneous redistribution at constant saturation). The efficiency of conversion of work to surface energy, $E_{d}$, depends on the increase in surface area that accompanies drainage. Surface areas of non-wetting phase/solid and nonwetting phase/wetting phase have been determined through displacement of a colored lowviscosity liquid resin which can be solidified so that thin sections reveal the distribution of phases and surfaces within the pore space of the rock. Two dimensional images obtained from thin sections were analyzed using stereology to obtain estimates of saturations and interfacial areas in three dimensions. For drainage of Berea sandstone to $20 \%$ wetting phase saturation, $E_{d}$ was $36 \%$ which was less than one half of the efficiency of $85 \%$ for the same range of change in saturation determined previously for random packings of equal spheres. Values of $E_{d}$ for the tested carbonate were about one half of those for sandstone. The wide variation is explained in terms of a simple pore model that relates $E_{d}$ to aspect ratio.
\end{abstract}




\section{Introduction}

Change in fluid saturations during multiphase displacements in porous media are accompanied by changes in interfacial surface area between the phases. Interfacial areas are directly related to surface energy and are fundamental to spontaneous imbibition phenomena, to multiphase transport properties such as relative permeability, and, to processes that involve mass transfer between phases. The relationship between work of displacement from capillary pressure data to changes in surface energy from direct measurements of surface areas has been reported in detail for drainage, imbibition, and secondary drainage for random packings of equal spheres. ${ }^{1}$ The first measurements of relationships between work and increase in surface energy for porous rocks are reported here for primary drainage of a sandstone and a limestone.

\section{Theory}

Capillary pressure data for porous media exhibit hysteresis between drainage and imbibition, basically because of capillary instabilities that accompany saturation change. The concepts of quasi-static displacement that have often been applied to obtain direct relationships between work of displacement, derived from the area under a capillary pressure curve, and change in surface free energy are therefore inapplicable. Justification for interpreting the area under capillary pressure curves as work of displacement has been presented. ${ }^{1}$ In fine detail, the curves were interpreted as a series of reversible displacements (termed isons) linked by spontaneous redistributions at constant saturation (termed rheons). Demonstration of this mechanistic interpretation has been provided for bead packs ${ }^{1}$ and for porous rocks by low-rate constant-flow mercury porosimetry, referred to as APEX, Apparatus for Pore Examination. ${ }^{2}$

The external work, $W$, needed to reduce the saturation of wetting phase, $\alpha$, of a porous solid with bulk volume of $V_{b}$ and porosity, $\phi$, from $100 \%$ to a saturation $S_{\alpha}$ is given by:

$$
W_{100, S_{a}}=V_{b} \phi \int_{100}^{S_{\alpha}} P_{c} d S_{\alpha}
$$

Where, $P_{c}$ is the capillary pressure.

The change in surface free energy, $\Delta F$, is a function of the interfacial area created by the invading non-wetting phase:

$$
\Delta F=\sigma_{\alpha \beta}\left(\Delta A_{\alpha \beta}+\Delta A_{\beta \gamma} \cos \theta\right)
$$

where, $\sigma_{\alpha \beta}$ is the interfacial tension, $\mathrm{mN} / \mathrm{m}, \sigma$ is the contact angle, $\beta$ is the non-wetting phase, $\Delta A_{\alpha \beta}$ is the interfacial area between the wetting and non-wetting phases, $\Delta A_{\beta \gamma}$ is the interfacial area between the non-wetting, $\beta$, and solid phase, $\gamma$.

If $\theta$ is zero, the increase in surface free energy that accompanies drainage is given by

$$
\Delta F=\sigma_{\alpha \beta}\left(\Delta A_{\alpha \beta}+\Delta A_{\beta \gamma}\right)
$$


Because of inherent instabilities in the drainage process, the increase in surface free energy is less than the work done. The net efficiency $(\%), E_{d}$, of conversion of work to surface energy for primary drainage to saturation, $S_{\alpha}$, is given by

$$
E_{d}=\frac{\sigma_{\alpha \beta}\left(\Delta A_{\alpha \beta}+\Delta A_{\beta \gamma}\right)}{V_{b} \phi \int_{100}^{S_{\alpha}} P_{c} d S_{\alpha}} 100
$$

\section{Rock Characterization}

Berea sandstone (referred to as Berea PH 2) from Cleveland Quarries, Ohio, and an Edwards limestone, referred to as Edwards GC, from near Garden City, Texas, were obtained in the form of 6"X12"x12" blocks. Values of porosity, permeability, BET surface area, and XRD clay analysis (sandstone only) are included in Table 6-1.

Examples of thin sections for the sandstone PH 2 are shown in Figs. 1a and b and SEMs in Figs. 6-1c and d. The matrix mainly consists of quartz grains, and includes lesser fractions of rock fragments, and partially dissolved feldspars. The SEMs show kaolinite and partially dissolved feldspar grains in detail.

The Edwards (GC) sample was composed of calcite minerals (Figs. 6-2a and b) with pore space consisting of mainly of moldic pores (clearly derived from dissolution of fossils) and interparticle porosity. The original interparticle porosity had been reduced significantly by recrystallization of calcite both within and outside these pores. The SEMs (Figs. 6-2c and d) clearly show that moldic pores have been partially refilled through re-crystallization of calcite.

Mercury injection capillary pressure data for Berea PH 2 sandstone and Edwards GC Block 2 limestone are shown in Figs. 6-3a and 6-3b respectively. Respective pore size distributions, shown in Fig. 6-3c and 6-3d were derived from a combination of mercury injection and desorption isotherm measurements (see Fig. 6-3c for the sandstone PH 2 and Fig. 6-3d for the limestone (Edwards GC Block 2)). The portion of the pore size distribution shown as a dashed curve in Fig. 6-3d, was estimated because the mercury injection pressure cell was changed during the course of the test.

\section{Experimental Procedures: Capillary Pressure and Interfacial Areas}

\section{Capillary pressure measurements for primary drainage}

Primary drainage capillary pressure measurements were made with a Beckman J 6B centrifuge. Cores of $3.81 \mathrm{~cm}(1.5 \mathrm{inch})$ diameter and up to $6 \mathrm{~cm}(2.36 \mathrm{inch})$ in length were first saturated with brine. Each core was then set in a sleeve which fitted into an Exxon core holder. A confining pressure of 15 bars (220 psi) was applied. For the sandstone, only displacement of brine by air was measured. For the carbonate sample (Edwards GC Block 2), displacement of brine by air and brine by decane (IFT against brine as supplied was $35 \mathrm{mN} / \mathrm{m}$ ) were measured. The capillary pressure depends on the angular speed of rotation, $\omega$, and the density difference, $\Delta \rho$, between the two fluids and is given by

$$
P_{c}=\frac{\omega^{2} \Delta \rho\left(r_{o}^{2}-r^{2}\right)}{2}
$$


where, $r_{o}$ is the distance from the centre of rotation to the outermost face of the core and $r$ is the distance from the centre of rotation to any point in the core. The average saturation, $S_{\alpha}(r, \omega)$, of the core is given by

$$
S_{\alpha}(r, \omega)=\frac{1}{L \pi R_{c}^{2}} \int S_{r, \omega} d V_{c}
$$

where, $L$ is the length of the core, and $R_{c}$ and $V_{c}$ are the radius and volume respectively.

Various methods of obtaining solutions to Eq 6-6 that involve converting the volume of fluid produced at any speed to saturation at the inlet face of the core, have been proposed. In a survey conducted by the Society of Core Analysts, Forbes et al. compared 19 different solutions to Eq. 6-6 and concluded that the final capillary pressure curve varied quantifiably from one method to another. ${ }^{3}$ In the present work, the Cubic Spline and the Ruth-Wong $1^{\text {st }}$ method ${ }^{4,5}$ were used for the sandstone, and the $2^{\text {nd }}$ Bentsen Version 2 was used for the carbonates. ${ }^{6}$ These methods were selected based on experience with respect to the fit to the raw data and the form of the derived capillary pressure data.

\section{Interfacial areas}

The following procedure was used to visualize the fluid distribution and interfacial areas that develop in a core under primary drainage. First, the core was saturated with a low viscosity resin containing a blue dye using both vacuum and high pressure in consecutive steps. Second, the fully saturated sample was centrifuged (air displacing the blue-dyed epoxy) to a particular net saturation at a selected speed for $72 \mathrm{hrs}$. The temperature in the centrifuge was then raised so that the epoxy resin became solidified in-situ with the core still spinning. The core was then placed in a pressure vessel and the air was evacuated followed by forcing red-dyed resin into the sample at a final pressure of $1500 \mathrm{psi}$. The second resin was then solidified by raising the temperature. Thin sections prepared from the core showed the areas of occupancy by the blue and the red (nominally) epoxy resins, which corresponded to the location of the wetting phase and the nonwetting phase respectively (Figs. 6-4a and b). Further details of the procedure are available.

\section{Image Analysis}

Optical images obtained with a petrographic microscope under reflected light provided satisfactory approximation to 2D images. The images were processed to obtain a description with only three types of pixels, blue, red and white, that represented the wetting, $\alpha$, non-wetting phase, $\beta$, and the solid phase, $\gamma$, respectively. A grid was overlain on the processed image and point counts and line intercepts were measured using in-house software. The point counts and line intercepts in conjunction with theorems of stereology ${ }^{8}$ provided the saturations and surface-tovolume ratios of the three phases. ${ }^{7}$

For the sandstone, a total of 85 micrographs were taken from 14 different thin sections made from 5 different cores. The results for an individual micrograph were averaged. 7 thin sections were evaluated at $10 \mathrm{X}$ resolution whereas the rest were analyzed at $20 \mathrm{X}$ as a check that values were not biased by the scale of measurement. The pixel sizes at $20 \mathrm{X}$ and $10 \mathrm{X}$ magnification were 0.74 and 2.04 microns respectively (the pixel size did not vary linearly with magnification 
because different microscope/camera combinations were used for each of the magnifications). The size of each picture so obtained was $0.08 \mathrm{~cm}^{2}$ at $10 \mathrm{X}$ magnification which was only about $1 / 140$ of the area of cross section of each thin section. Scatter in the data is to be expected because of the small area sampled for each image. A 1 X1 pixel grid (the finest grid possible) was used for analysis.

The fundamental assumption of stereology is that the 2D image is representative of the 3D sample. Agreement in global properties, such as porosity (3D property) obtained from thin sections and that obtained volumetrically, provided indication that the micrographs provided satisfactory resolution. If, for a particular thin section, the variation in global properties was large from one micrograph to another and also from that obtained volumetrically, then a lower magnification was used. For example the use of $40 \mathrm{X}$ magnification leads to large variation in porosity between images because of the small area scanned at this magnification. For the data presented, magnifications of $10 \mathrm{X}$ and $20 \mathrm{X}$ gave close values of average porosity (17.1 and $16.9 \%$ ). These values were consistent with respect to the volumetrically determined values of $21 \%$ because fine pore structure cannot be resolved by petrographic analysis.

For the limestone, one core was cut from Edwards GC limestone Block 1 and the other from Block 2. 9 thin sections (5 from Block 1 and 4 from Block 2) were prepared. A total of 57 micrographs were analyzed (22 from Block 1 and 35 from Block 2 ) at $20 \mathrm{X}$ magnification. The average porosity of $20.5 \%$ from the thin sections was consistent with the volumetrically measured value of $23 \%$.

Three interfacial areas expressed in $\mathrm{m}^{2} / \mathrm{g}$, wetting phase/solid, $A_{\alpha \gamma}^{*}$, non-wetting phase/solid, $A_{\beta \gamma}^{*}$, and wetting/non-wetting phase, $A_{\alpha \beta}^{*}$, were derived from the micrographs. Two additional areas, the total solid surface area, and the created surface area, also expressed as $\mathrm{m}^{2} / \mathrm{g}$ of dry rock were defined by these areas. Total solid surface area, $\sum A_{\gamma}^{*}$, was operationally defined by the imaging procedure. For the images that contained resins of two colors, $\sum A_{\gamma}^{*}$ was obtained from the sum of the interfacial areas between the solid-red, $A_{\gamma}^{*}$, and the solid-blue, $A_{\alpha \gamma}^{*}$, interfaces. The values of $\sum A_{\gamma}^{*}$ provided indication that the solid surface areas derived from petrographic analysis of the either the blue or red/blue resin impregnated thin sections were consistent. Ideally, $\sum A_{\gamma}^{*}$ should be constant. The created surface area, $\sum A_{\beta}^{*}$, associated with invasion of the non-wetting phase, was given by the sum of the solid-red, $A_{\beta \gamma}^{*}$, and the red-blue, $A_{\alpha \beta}^{*}$, surface areas.

\section{Results and Discussion}

\section{Work of primary drainage from capillary pressure curves}

Raw centrifuge data and derived drainage capillary pressure curves by three methods for displacement of brine by air are shown in Fig. 6-5a for Berea sandstone PH 2. The work of displacement per gram of dry solid, $W^{*}$, obtained by integrating under the capillary pressure curve is shown in Fig. 6-5b. Results for two Edwards GC limestone cores cut from Block 2, one for displacement of brine by air, and the other for brine by decane, are presented in Figs. 6a and $6 \mathrm{~b}$ respectively. 
After scaling the capillary pressure for difference in interfacial tension, the curvature, $P_{c} / \sigma_{\alpha \beta}$, for air/brine was higher than oil/brine. Also, displacement curvatures for mercury injection (given by scaling the capillary pressure data shown in Figs. 6-3a and $\mathrm{b}$ with respect to interfacial tension and the cosine of contact angle for either $0^{\circ}$ or $40^{\circ}$ ) did not scale consistently with either air/brine or oil/brine centrifuge data. Relationships between work of displacement and increase in surface free energy are summarized in Table 6-2. Many examples of inconsistency between capillarypressure data sets have been reported previously. ${ }^{9-15}$ The most complete capillary pressure data sets obtained for rocks used in the present work were for displacement of brine by air; analysis is mainly based on these data.

\section{Total Solid $\sum A_{\gamma}^{*}$ and Created $\sum A_{\beta}^{*}$ Surface Areas}

\section{Sandstone}

The average value of $\sum A_{\gamma}^{*}$, (see Fig. 6-7a) of about $0.01 \mathrm{~m}^{2} / \mathrm{g}$, was operationally defined by the imaging procedure. $\sum A_{\gamma}^{*}$ was almost two orders of magnitude less than the area of $0.626 \mathrm{~m}^{2} / \mathrm{g}$, operationally defined by gas adsorption, because the optical imaging does not resolve details of the surfaces of clays and other forms of microporosity that are the major contributors to the BET surface area.

The interfacial area between the wetting and the non-wetting phase, $A_{\alpha \beta}^{*}$, was never more than $2 \%$ of $\sum A_{\gamma}^{*} \cdot \sum A_{\beta}^{*}$ (the sum of $A_{\beta \gamma}^{*}$ and $A_{\alpha \beta}^{*}$ ) increased close-to-linearly with the decrease in the wetting phase saturation (see Fig. 6-7b).

\section{Carbonate}

For Edwards GC limestone Blocks 1 and 2, solid surface areas determined from imaging, $\sum A_{\gamma}^{*}$, were respectively $0.0146 \mathrm{~m}^{2} / \mathrm{g}\left(7.3 \%\right.$ of BET surface area) and $0.0133 \mathrm{~m}^{2} / \mathrm{g}(3.3 \%$ of BET surface area) (see Fig. 6-8a). As for sandstone, created surface area, $\sum A_{\beta}^{*}$, increased linearly with decrease in wetting phase saturation for both samples (Fig. 6-8b). The interfacial area between the wetting and the non-wetting phase, $A_{\alpha \beta}^{*}$, exhibited maxima that were only $5 \%$ and $2 \%$ of, $\sum A_{\gamma}^{*}$, for Blocks 1 and 2 respectively (Fig. 6-8b).

\section{Efficiency of Conversion of Work to Surface Energy Sandstone, $E_{d}$}

The work of displacement per gram of dry solid, $W^{*}$, obtained from air/brine centrifuge data, expressed as an equivalent area $W^{*} / \sigma_{\alpha \beta}$, and the changes in created surface area, $\sum A_{\beta}^{*}$, obtained from thin sections for Berea sandstone PH 2 are shown in Fig. 6-9a. The efficiency of conversion of work to surface energy decreases with increase in wetting phase saturation (see Fig. 6-9b). For the sandstone $\mathrm{PH} 2$, the efficiency, $E_{d}$, of primary drainage to $20 \%$ wetting phase saturation was $36 \%$. 


\section{Carbonate, $E_{d}$}

$W^{*}$ for Edwards GC Block 2 obtained from air/brine centrifuge data, expressed as an equivalent area, is shown in Fig. 6-10a. Changes in created surface area, $\sum A_{\beta}^{*}$, obtained from thin sections for the Edwards GC Blocks 1 and 2, are also shown. The efficiency of conversion of work of primary drainage (down to $\mathrm{S} \alpha=20 \%$ ) to surface energy for Block 2 was only $14 \%$ (see Fig. 610b). Air/brine capillary pressure data for Block 1 were not available. Based on the air/brine capillary pressure data for Block $2, E_{d}$ for Block 1 was $18 \%$ (see Fig 6-10b). The average air/brine-based value of $E_{d}$ of $16 \%$ is used for limestone in further discussion.

\section{Random packings of equal spheres}

Random packings of equal spheres provide a readily prepared class of porous media with statistically similar geometry and porosity that is usually close to $37 \%$. The permeability of the bead pack is proportional to the square of the sphere radius, R. Provided the ratio of gravity to capillary forces is not unusually high, and that spreading prevails $(\theta=0)$, capillary pressures measurements for drainage, imbibition, and secondary drainage should give universal plots of dimensionless pressure $\left(P_{c} R / \sigma_{\alpha \beta}\right)$ versus saturation.

A characteristic feature of drainage of sphere packs, which results from smooth surfaces, is that part of the wetting phase is retained as hydraulically disconnected capillary structures, the most well-known example being pendular rings at point contacts between spheres. The capillary pressure and hence the surface curvature of each individual capillary structure, or the pressure acting at a semi-permeable membrane is set at the time of isolation from the continuous part of the wetting phase. ${ }^{16}$ The isolated structures of the wetting phase are not in capillary equilibrium with each other or the externally measured pressure (this might be the hydrostatic head for a packed column or the capillary pressure at a semi-permeable membrane). However, the times required to attain thermodynamic equilibrium by diffusion or transport in surface films are many orders of magnitude greater than the times required to reach mechanical equilibrium. The fraction of wetting phase that is retained as an irreducible saturation with respect to mechanical equilibrium is very close to $7 \%$ for any size of sphere.

The fraction of drained sphere surface at the irreducible saturation was determined by drainage of dilute acid from packings of ball bearings followed by point counting of etched and nonetched areas. For any sphere size, the fraction of drained sphere surface was constant and equal to $71 \%$ of the total sphere surface. The wetting/non-wetting surface area at the irreducible saturation was equal to about $7.5 \%$ of the total sphere surface area. From these values, and the the work given by the universal curve for drainage to the $7 \%$ irreducible wetting phase saturation, $E_{d}$ was $79 \%{ }^{1}$

$E_{d}$ for sphere packs, as found for the sandstone and carbonate, also decreased with increase in wetting phase saturation (see Fig. 6-11). For drainage of random packings of equal spheres to $20 \%$ saturation, Ed was $85 \%$. The values of $E_{d}$ for air/brine drainage of Berea PH 2 sandstone and Edwards limestone down to $20 \%$ wetting phase saturation are $36 \%$ and $16 \%$ respectively. The capillary pressure data and surface areas obtained for rocks involve much greater uncertainties than for sphere packs. However, even for the range of values given by the different conditions of measurement of capillary pressure data, values of $E_{d}$ for the sandstone and limestone (see Table 6-2) are always much lower than for spheres. 


\section{Effect of Aspect Ratio on $\boldsymbol{E}_{d}$}

If the work of displacement in moving an interface is reversible, as in displacement in a cylindrical tube, the efficiency of conversion of work to surface free energy is $100 \%$. Loss of surface free energy during drainage of porous media is caused by capillary instabilities. For example, consider a pore consisting of a spherical cavity of radius, $R_{s p}$, with throat radius, $r_{t}$, (see Fig. 6-12a).

The length, $L_{t}$, of a cylindrical tube of radius $r_{t}$ having the same volume as the sphere of radius $\left(R_{s p}\right)$ is given by:

$$
\pi r_{t}^{2} L_{t}=\frac{4}{3} \pi R_{s p}^{3}
$$

and,

$$
L_{t}=\frac{4 R_{s p}^{3}}{3 r_{t}^{2}}
$$

The capillary pressure needed to drain the spherical cavity is set by the pore throat size. At this pressure, the interface becomes unstable and passes through the cavity. The ratio of the area created in draining the spherical cavity (we assume that upon drainage the cavity becomes filled by the non-wetting phase and, as a first approximation, neglect the area of the sphere associated with its throats) to that for reversible drainage of the equivalent cylindrical pore gives the efficiency, $E_{d}$, of conversion of work to surface free energy.

$$
E_{d}=\frac{4 \pi R_{s p}^{2}}{2 \pi r_{t} L_{t}}
$$

Substituting for $L_{t}$, the efficiency, $E_{d}$, is given by

$$
E_{d}=\frac{1.5 r_{t}}{R_{s p}}
$$

If the ratio of pore body to throat radius, commonly referred to as the aspect ratio, is denoted as $\xi$,

$$
E_{d}=\frac{1.5}{\xi}
$$

Results obtained for displacement efficiencies as a function of the aspect ratio given by Eq. 6-11 $\left(\theta=0^{0}\right)$ are shown in Fig. 6-12b. The efficiency of drainage is highly sensitive to the ratio of pore throat to pore body size. In addition to aspect ratio, the accessibility of individual pores will contribute to the inefficiency of drainage. The magnitude of the sudden pressure changes measured by Yuan $^{2}$ indicates that drainage of pores often occurs in large clusters. Then, the pressure of drainage may not be set by the penetration pressure of the pore throat of an individual 
pore but by some other pore throat that controls access and only empties at higher capillary pressure. In effect, this increases the work of displacement for such pores.

Aspect ratios have also been related to snap-off and the tendency for non-wetting phase to become trapped during imbibition processes at very strongly water wet conditions. ${ }^{17}$ For cubic and hexagonal packings of spheres, the aspect ratios are 1.45 and 2.67 respectively, based simply on in spheres for the pore throats and bodies.

The reported residual oil for random sphere packs is only $15.5 \% .{ }^{18}$ For Berea sandstone, the aspect ratio from the data presented by Wardlaw et al. ${ }^{19}$ was 7.7 and from Chatzis et al. ${ }^{20}$ the aspect ratio ranged from $4-6.7$. The residual saturation to non-wetting phase for very strongly water-wet Berea sandstone is typically about $50 \%$ of the initial oil saturation. For the Edwards GC carbonates, the range of aspect ratio can be seen from the moldic pore sizes, indicated by SEM and micrographs of thin sections (Figs. 6-2c and d), versus the throat sizes, indicated by the mercury injection capillary pressures for drainage (Fig. 6-3b), to be very high. Trapping of nonwetting phase for the Edwards GC limestone used in the present work was about $65 \%{ }^{21}$

\section{Aspect Ratio and Wettability}

If the contact angle for the pore model of Fig. 6-12a is $\theta$, the surface energy of the drained cavity is given by

$$
4 \pi R_{s p}^{2} \cos \theta
$$

If the drainage pressure is proportional to the cosine of the contact angle, as for a cylindrical tube, then $E_{d}$ is independent of wettability. However, if the drainage pressure of the cavity is insensitive to contact angle, because of factors, such as edges, surface roughness, and converging-diverging pore shapes, that result in low effective receding contact angles, ${ }^{22,23}$ the efficiency is given by

$$
E_{d}=\frac{1.5 \cos \theta}{\xi}
$$

Drainage efficiencies can then be very low. On the other hand, if a significant fraction of the bulk wetting phase is retained by surface roughness, microporosity, and macro-sized pore throats, at otherwise drained pore surfaces, values of $E_{d}$ will be correspondingly higher.

\section{Nomenclature}

A interfacial area, $\mathrm{m}^{2}$

$A^{*} \quad$ interfacial area, $\mathrm{m}^{2} / \mathrm{g}$ of dry rock

$\sum A_{\gamma}^{*} \quad$ total solid surface area, $\mathrm{m}^{2} / \mathrm{g}$ of dry rock

$\sum A_{\beta}^{*} \quad$ created surface area, $\mathrm{m}^{2} / \mathrm{g}$ of dry rock

$A_{w}^{*} \quad$ surface area equivalent to scaled work of displacement, $W^{*} / \sigma, \mathrm{m}^{2} / g$

$E_{d} \quad$ efficiency (\%) of conversion of work of drainage to surface free energy

$K \quad$ permeability of the core sample, md

$L \quad$ length of tube or core, $\mathrm{cm}$

$P_{c} \quad$ capillary pressure, $\mathrm{KPa}$ 


$\begin{array}{ll}R, r & \text { radius, cm } \\ V & \text { bulk volume, } \mathrm{cm}^{3} \\ W & \text { work of drainage, } K J \\ W^{*} & \text { work per gm of dry rock, } \mathrm{KJ} / \mathrm{g} \\ \theta & \text { contact angle, degrees } \\ \xi & \text { aspect ratio } \\ \rho & \text { density of the fluid, } \mathrm{g} / \mathrm{cm}^{3} \\ \sigma & \text { interfacial tension, } \mathrm{mN} / \mathrm{m} \\ \phi & \text { porosity } \\ \omega & \text { angular speed of rotation, } \mathrm{rad} / \mathrm{sec}\end{array}$

Subscripts:

$\begin{array}{ll}b & \text { bulk } \\ c & \text { core } \\ s p & \text { spherical } \\ t & \text { throat } \\ \alpha & \text { wetting phase } \\ \beta & \text { non-wetting phase } \\ \gamma & \text { solid phase }\end{array}$

\section{References}

1.Morrow, N.R.: "Physics and Thermodynamics of Capillary Action in Porous Media", Ind. and Eng. Chem. 62, No. 6, (1970), 32-56.

2.Yuan, H.H.: "Pore-Scale Heterogeneity From Mercury Porosimetry Data", SPEFE 14892 (March 1991), 17-24.

3.Forbes, P.: "Centrifuge Data Analysis Techniques: An SCA Survey on the Calculation of Drainage Capillary Pressure Curves from Centrifuge Measurements", SCA 9714 proceedings of 1997 International Symposium of the SCA, Calgary, Canada.

4.Ruth, D., and Wong, S.: "Calculation of Capillary Pressure Curves from Data Obtained by the Centrifuge Method", proceedings of 1988 International Symposium of the SCA, Houston, TX., Aug 17-18.

5.Ruth, D., and Wong, S.: "Calculation of Capillary Pressure Curves from Data Obtained by the Centrifuge Method", The Log Analyst, v. 32, n.5, (1991), p. 575-582.

6.Bentsen, R.G., and Anli, J.: "Using Parameter Estimation Technique to Convert Centrifuge Data into Capillary Pressure Curve”, SPE\#5026, SPEJ, Feb. (1977), p. 57-64.

7.Seth, S.: "Increase in Surface Energy by Drainage of Sandstone and Carbonate", Ph.D. Thesis, (2006), University of Wyoming, Laramie, WY, USA.

8.Underwood, E.E.: "Quantitative Stereology”, Addison-Wesley Publishing Company, Reading, MA, (1970).

9.Sallier, B., and Hamon, G.: "Micritic Limestone of the Middle-East: Influence of Wettability,

Pore Network and Experimental Technique on Drainage Capillary Pressure Curve", SCA 2005-08 proceedings of 2005 International Symposium of the SCA Toronto, Aug 21-25. 10. Purcell, W.R.: "Capillary Pressures - Their Measurement Using Mercury and the Calculation 
of Permeability There from", Petroleum Transactions, AIME, February, 1949, 39-48.

11. Sabatier, L.: "Comparative Study of Drainage Capillary Pressures Using Different Techniques and for Different Fluid System", SCA 9424 proceedings of 1994 International Symposium of the SCA Stavanger, Norway.

12. Morrow, N.R., Buckley, J.S., Cather, M.E., Brower, K.R., Graham, M., Ma, S., Zhang, X.: "Rock Matrix and Fracture Analysis of Flow in Western Tight Gas Sands", Final Report to the U. S. Department of Energy, (Feb 1990), pp. 155-169.

13. Melrose, J.C., Dixon, J.R., and Mallinson, J.E.: "Comparison of Different Techniques for Obtaining Capillary Pressure Data in the Low-Saturation Region", SPE 22690, 1991 SPE ATCE Dallas, (Oct. 6-9).

14. Wells, J.D., and Amaefule, J.O.: "Capillary Pressure and Permeability Relationships in Tight Gas Sands", SPE 13879, 1985 SPE/DOE Low Permeability Gas Reservoirs held in Denver, CO May 19-22.

15. Hamon G., and Pellerin, F.M.: "Evidencing Capillary Pressure and Relative Permeability Trends for Reservoir Simulation”, SPE 38898, 1997 SPE ATCE San Antonio, Oct. 5-8.

16. Morrow, N.R.: "Irreducible Wetting-phase Saturations in Porous Media", Chem. Eng. Sci., 25, (1970), 1799-1815.

17. Wardlaw, N.C., and Casson, J.P.: "Oil Recovery Efficiency and the Rock-pore Properties of some Sandstone Reservoirs", Bull. Can. Petr. Geol., June, 27, (1979), 117-138.

18. Morrow, N.R., Chatzis, I., and Taber, J.J.: "Entrapment and Mobilization of Residual Oil in Bead packs", SPERE, (August, 3, 1988), 927-934.

19. Wardlaw, N.C., Li, Y., and Forbes, D.: "Pore-throat size Correlation from Capillary Pressure Curves", Transport in Porous Media 2, (1987) 597-614.

20. Chatzis, I., Morrow, N.R., and Lim, H.T.: "Magnitude and Detailed Structure of Residual Oil Saturation," SPEJ, Apr. 1983, 35, 311.

21. Tie, H., and Morrow, N.R.: "Low Flood Rate Residual Saturation in Carbonate Rocks", IPTC\#10470, International Petroleum Technology Conference, Doha, Qatar, 12-23 Nov. 2005.

22. Morrow, N.R., and McCaffery F.G: "Fluid Displacement Studies in Uniformly Wetted Porous Media", Wetting, Spreading and Adhesion, Ed. J.F. Padday, Academic Press, New York, pp. 289-319, (1978).

23. Morrow, N.R.: "The Effects of Surface Roughness on Contact Angle with Special Reference to Petroleum Recovery", Journal of Canadian Petroleum Tech., Oct.-Dec. (1975).

Table 6-1 Rock properties

\begin{tabular}{|c|c|c|c|c|}
\hline Rock & Solid Density, g/cc & Porosity, $\%$ & Permeability, md & CEC, meq/100g \\
\hline Berea Sandstone PH 2* & 2.65 & 21 & 650 & 0.145 \\
\hline Edwards GC (Block 1) Limestone & 2.69 & 24 & $10-15$ & - \\
\hline Edwards GC (Block 2) Limestone & 2.69 & 24 & $20-30$ & 0.026 \\
\hline
\end{tabular}

* XRD clay ratios: chlorite; illite; kaolinite $-16 ; 27 ; 57$ 
Table 6-2 Comparison of efficiency of drainage $E_{d}$, obtained based on primary drainage capillary pressure curves obtained by different methods for sandstone and limestone

\begin{tabular}{|c|c|c|c|}
\hline Capillary pressure method & $\begin{array}{c}\mathrm{P}_{\mathrm{c}} \text { equivalent } \\
\text { area } \\
\mathrm{A}_{\mathrm{W}}^{*}, \mathrm{~m}^{2} / \mathrm{g}\end{array}$ & $\begin{array}{c}\text { Area from thin } \\
\text { section } \\
\sum \mathrm{A}_{\beta}^{*}, \mathrm{~m}^{2} / \mathrm{g}\end{array}$ & $\begin{array}{c}E_{d} \\
\%\end{array}$ \\
\hline Berea sandstone PH 2 & 0.0081 & 35.8 \\
\hline Centrifuge (air/brine) & 0.0226 & 0.0081 & 20.3 \\
\hline Mercury injection (Hg/air) & 0.03987 & 0.011 & 18.3 \\
\hline Edwards GC limestone & 0.06 & 0.0086 & 14.3 \\
\hline Centrifuge (air/brine) Block 1 & 0.06 & 0.0086 & 20.5 \\
\hline Centrifuge (air/brine) Block 2 & 0.042 & 0.0086 & 18.1 \\
\hline Centrifuge (oil/brine) Block 2 & 0.0475 & &
\end{tabular}

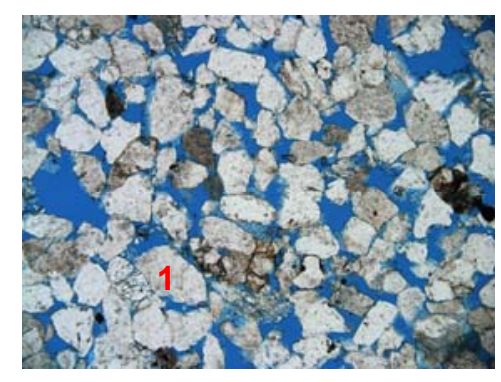

(a) Width $2320 \mu$

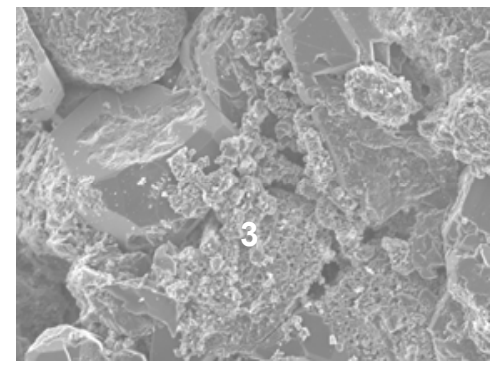

(c) Width $528 \mu$

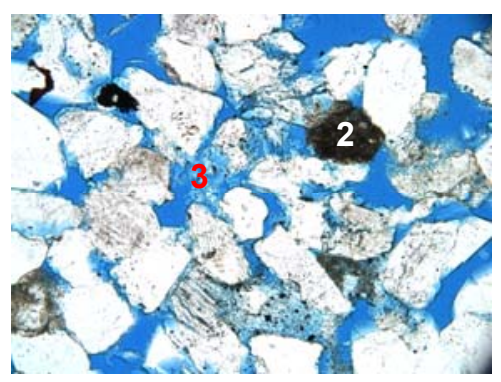

(b) Width $1186 \mu$

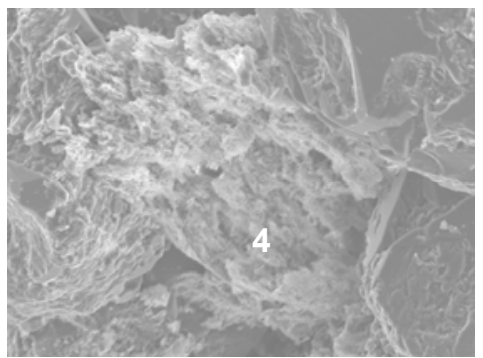

(d) Width $307 \mu$

Fig. 6-1 Features of Berea sandstone PH 2 from thin section, (a) and (b) and SEM, (c) and (d): quartz grain (1), rock fragments (2), kaolinite (3), partially dissolved feldspar (4). 


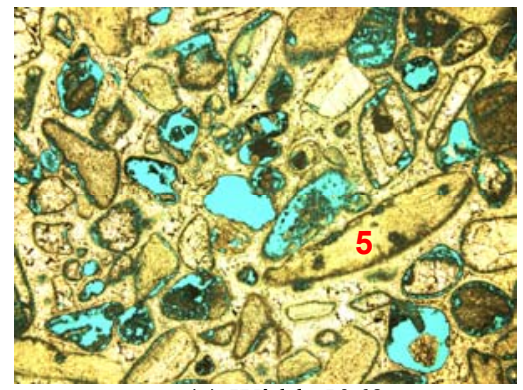

(a) Width $5968 \mu$

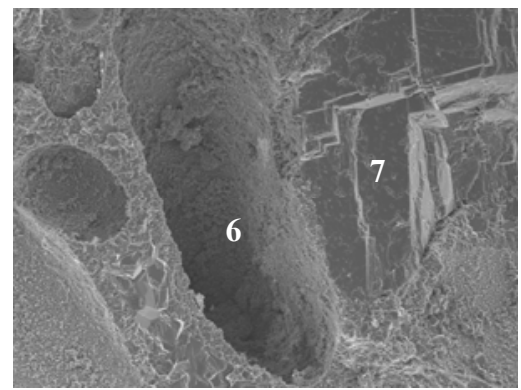

(c) Width $1000 \mu$

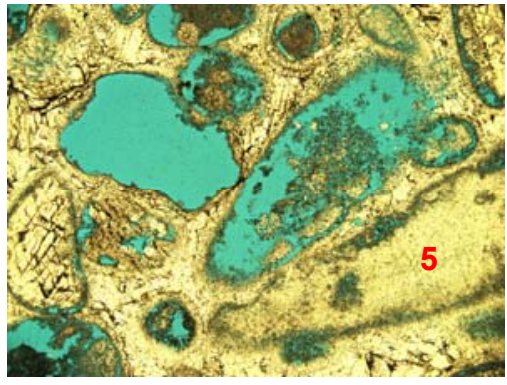

(b) Width $2320 \mu$

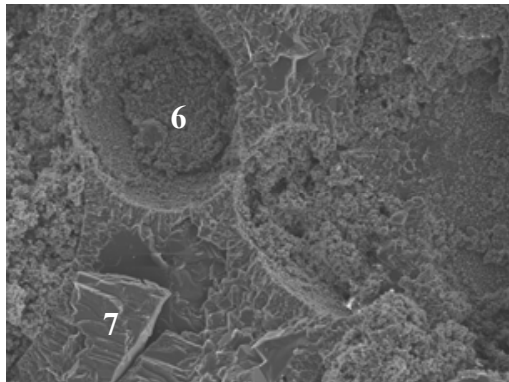

(d) Width $660 \mu$

Fig. 6-2. Features of Edwards GC Block 1 from thin section, (a) and (b), and SEM, (c) and (d): calcite grains (5); poorly connected moldic pores, (6) calcite cement (7).

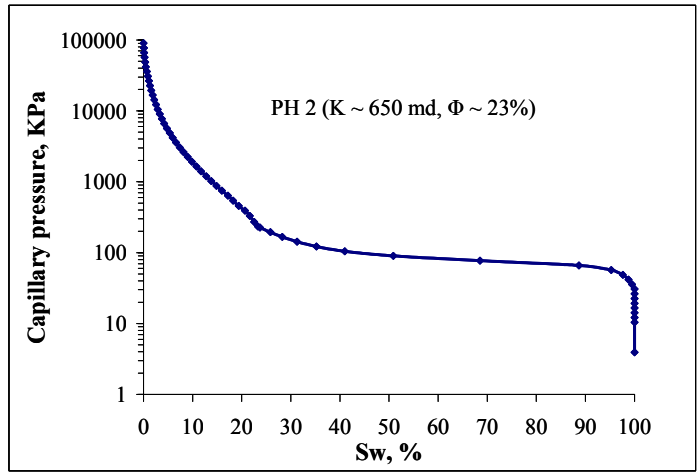

(a)

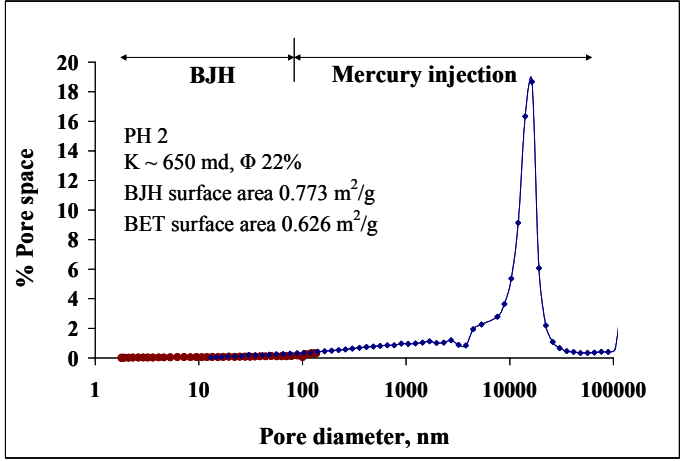

(c)

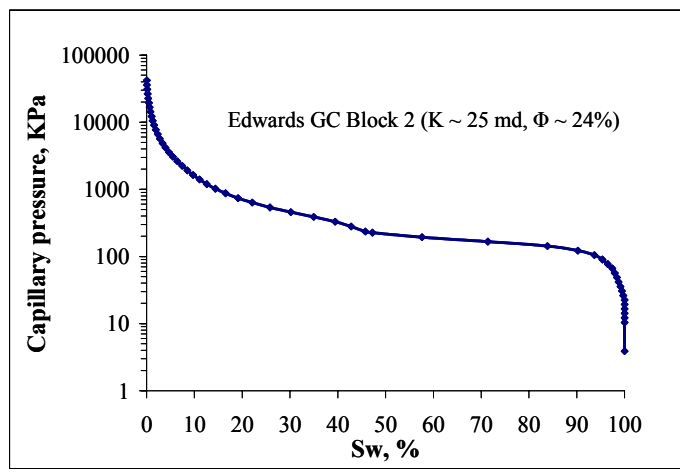

(b)

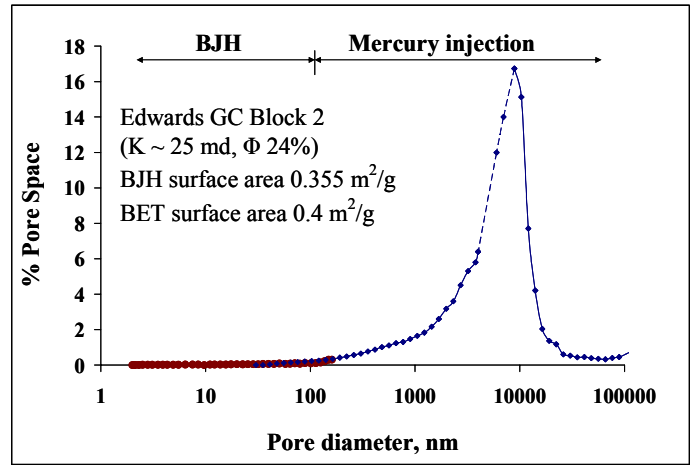

(d)

Fig. 6-3. Mercury injection capillary pressure curves for (a) Berea sandstone PH 2 (b) Edwards GC Block 2 limestone. Pore size distribution as a function of the pore volume for (c) sandstone (d) limestone. 


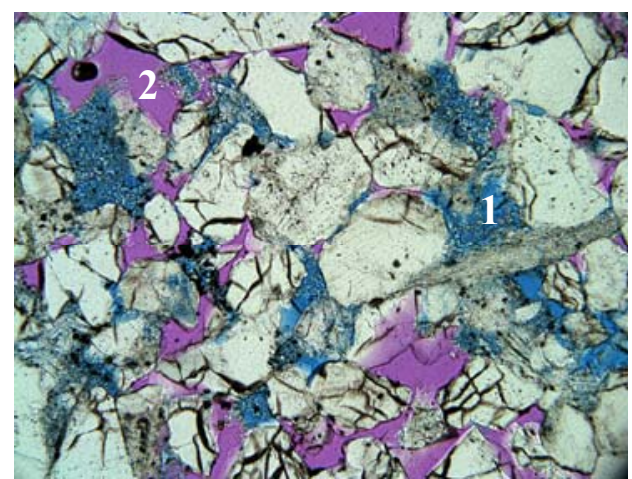

(a) Width $1186 \mu$

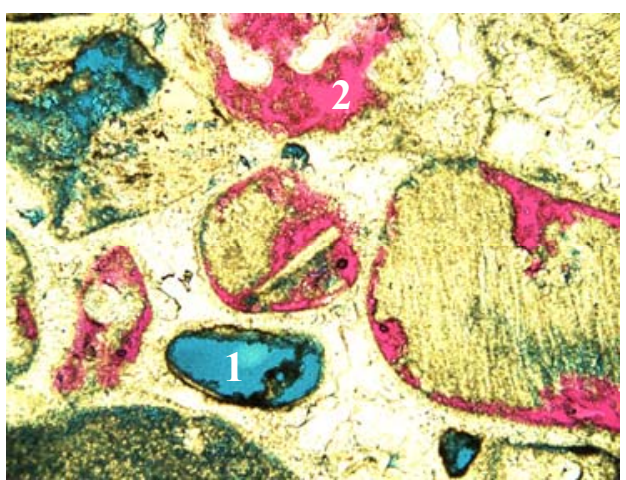

(b) Width $1186 \mu$

Fig. 6-4. Blue (wetting phase) (1), and red (non-wetting phase) (2), in: (a) Berea sandstone PH 2 at wetting phase saturation $S_{\alpha}=55 \%$; (b) Edwards GC limestone Block 2, wetting phase saturation, $S_{\alpha}=12 \%$.

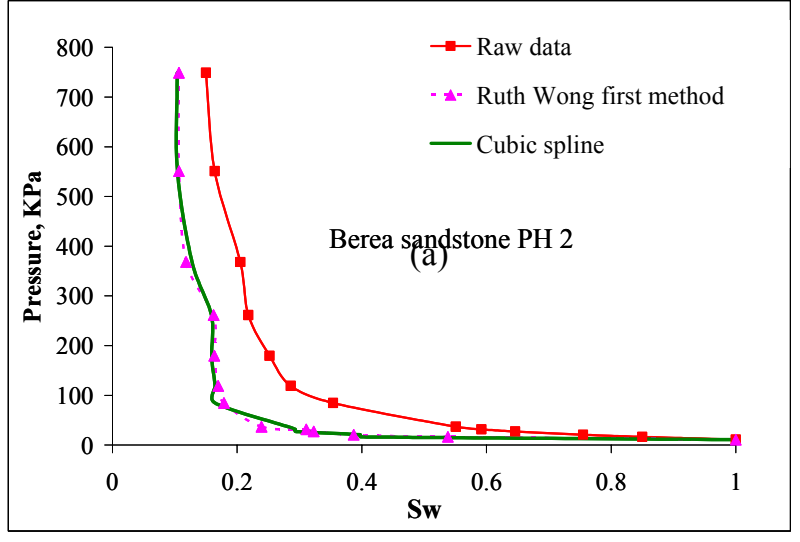

(a)

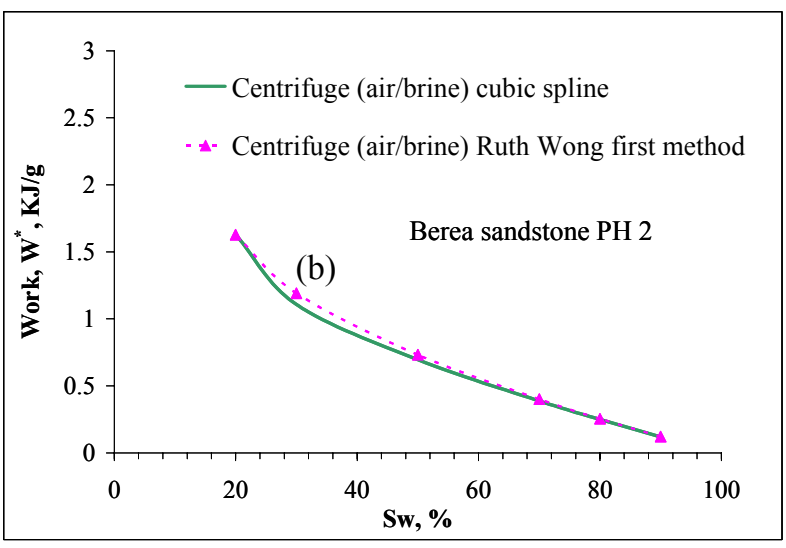

(b)

Fig. 6-5. (a) Capillary pressure curves derived by two examples of methods of converting raw centrifuge data to saturation at the inlet core face for sandstone (air-brine displacement). (b) Work of primary drainage per gram of dry solid, $W^{*}$, for sandstone obtained by integrating under the derived capillary pressure curves. 


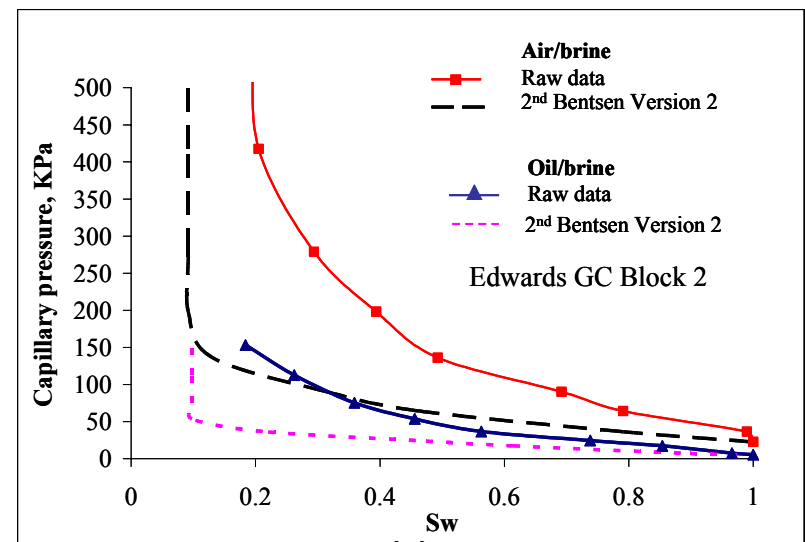

(a)

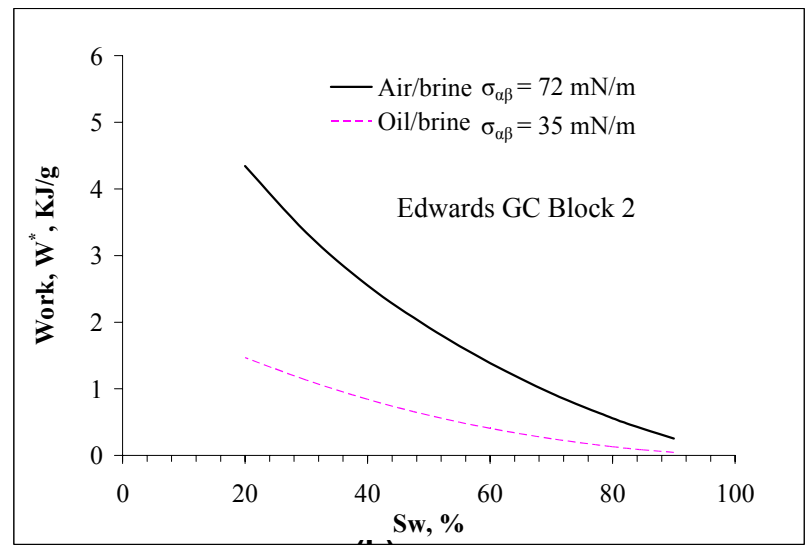

(b)

Fig. 6-6. (a) Capillary pressure curves derived by converting raw centrifuge data to saturation at the inlet core face for limestone. (b) Work of primary drainage per gram of dry solid, $W^{*}$, for limestone obtained by integrating under the derived capillary pressure curves.

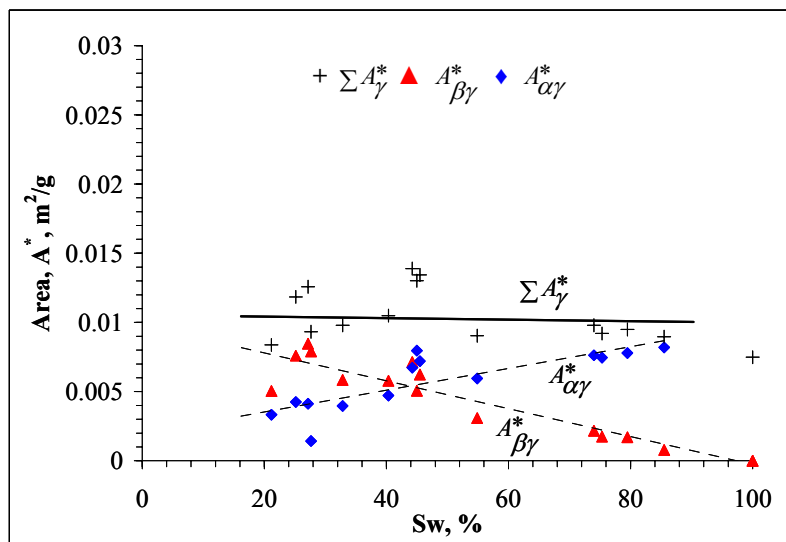

(a)

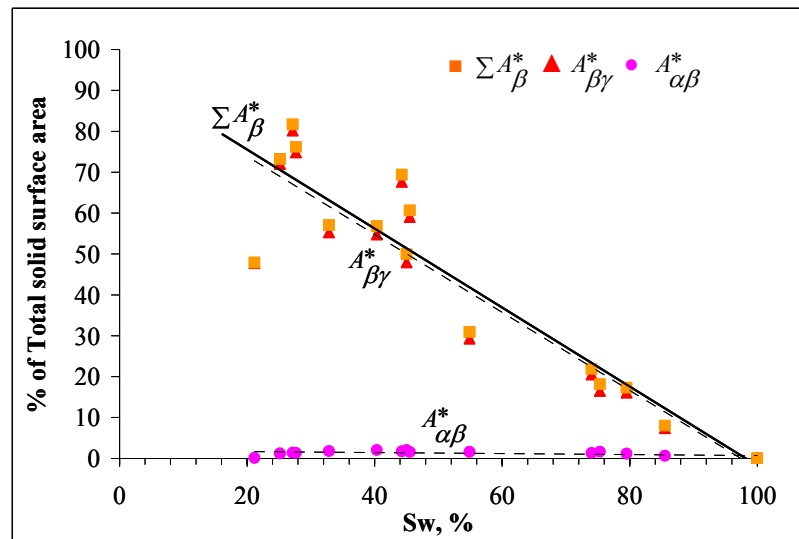

(b)

Fig. 6-7. Results from thin section analysis for Berea sandstone PH 2:

(a) total solid surface area, $\sum A_{\gamma}^{*} ;$ (b) created surface area, $\sum A_{\beta}^{*}$.

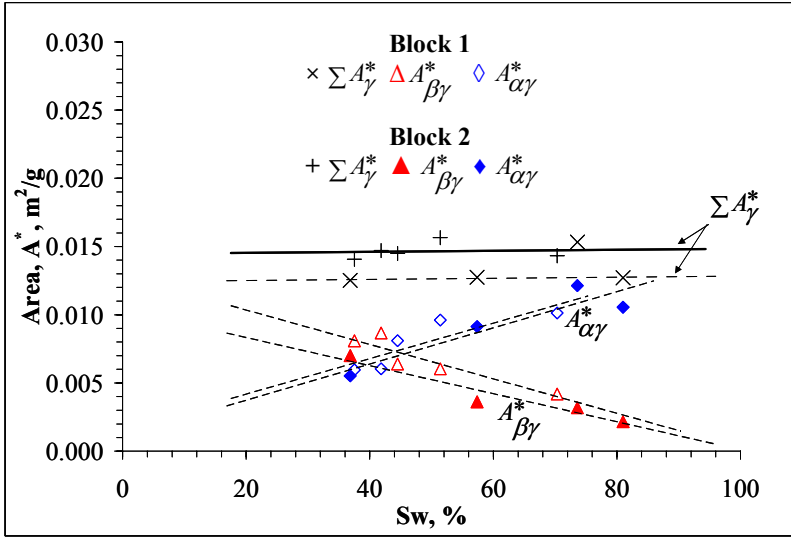

(a)

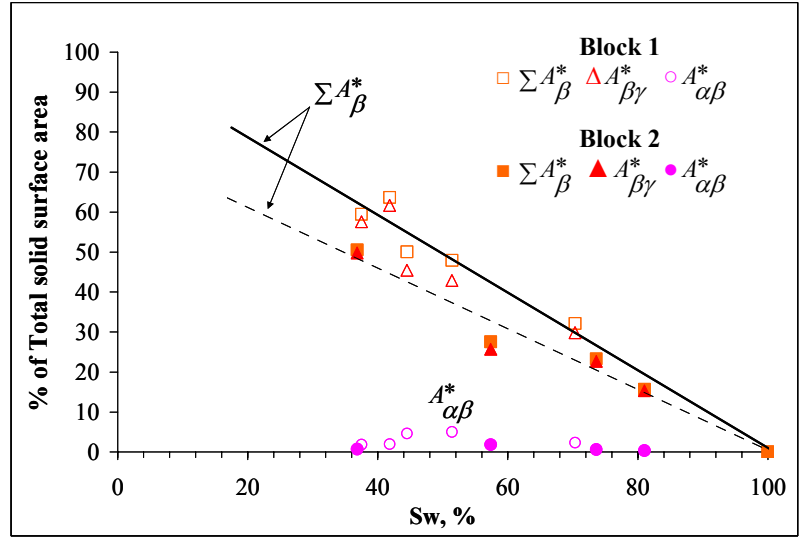

(b)

Fig. 6-8. Results from thin section analysis for Edwards GC limestone Blocks 1 and 2:

(a) total solid surface area, $\sum A_{\gamma}^{*} ;$ (b) created surface area, $\sum A_{\beta}^{*}$. 


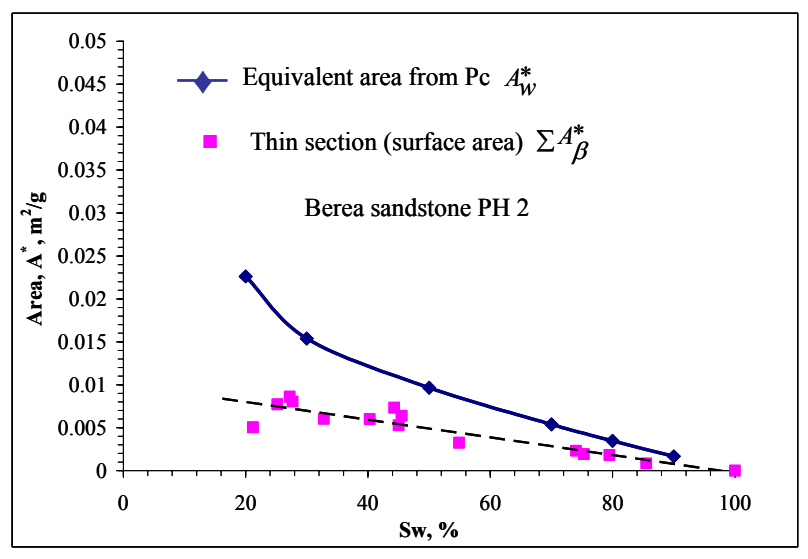

(a)

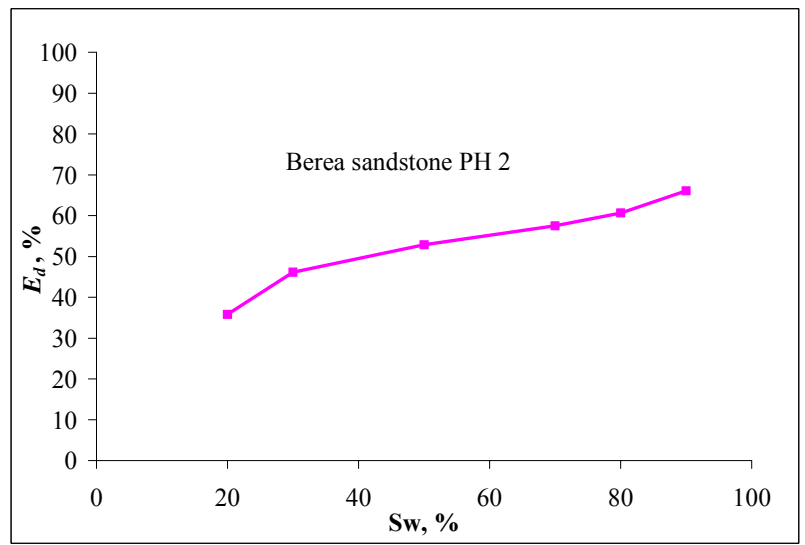

(b)

Fig. 6-9. (a) Equivalent area, $A_{w}^{*}$, equal to scaled work, $W^{*} / \sigma_{\alpha \beta}$, obtained by integrating under the capillary pressure curve (air/brine centrifuge) and the corresponding change in surface area, $\sum A_{\beta}^{*}$, obtained from thin section analysis. (b) Efficiency as a function of the wetting phase saturation for the sandstone.

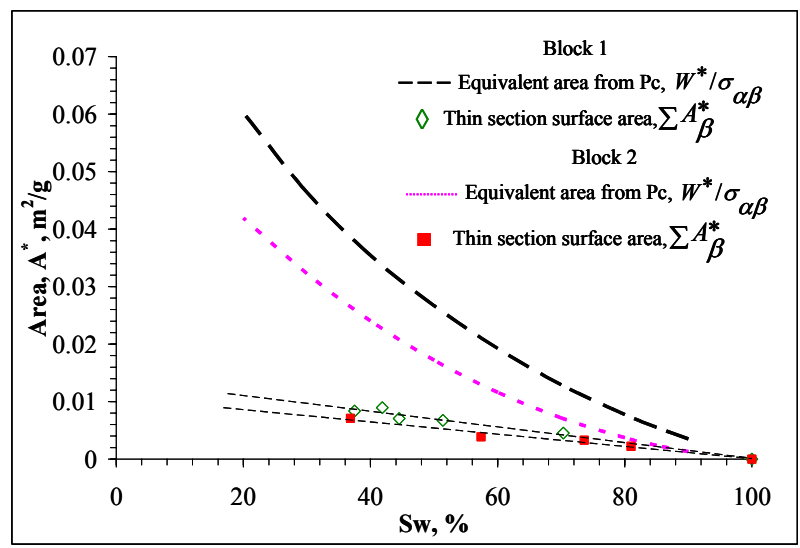

(a)

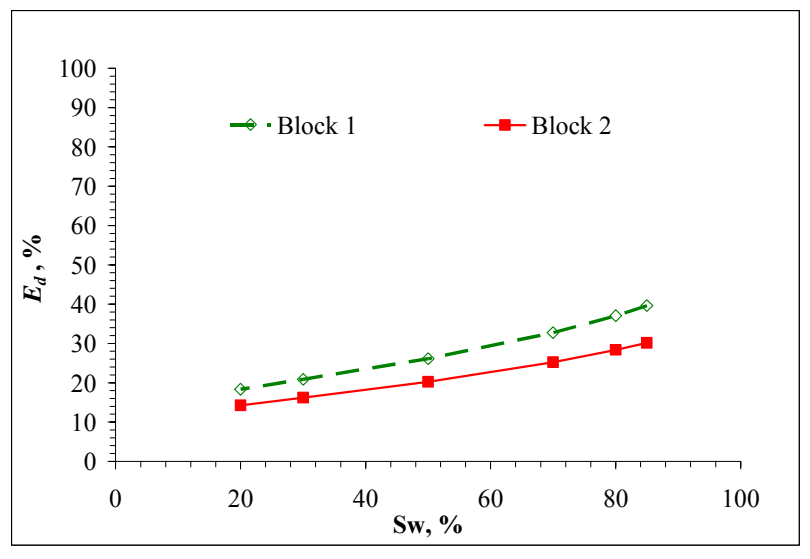

(b)

Fig. 6-10 (a) Comparison of equivalent area, $A_{w}^{*}$, equal to scaled work done $\left(W^{*} / \sigma_{\alpha \beta}\right)$ obtained by integrating under the capillary pressure curve (air/brine and oil/brine centrifuge) and the corresponding change in surface area, $\sum A_{\beta}^{*}$, obtained from thin section analysis for Edwards GC limestone Blocks 1 and 2. (b) Efficiency as a function of the wetting phase saturation for the limestone based on air-brine centrifuge results for Block 2. 


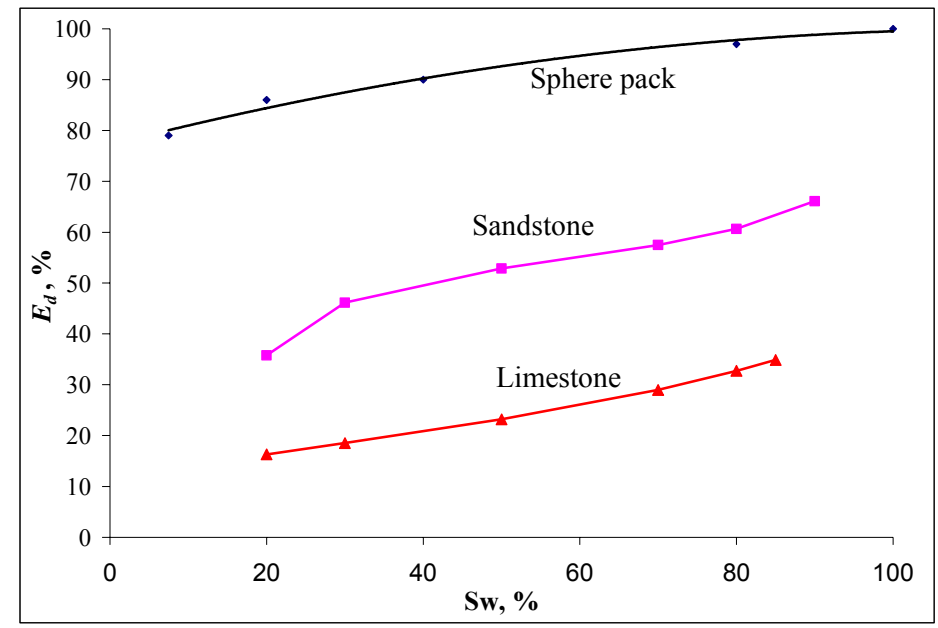

Fig. 6-11. Comparison of efficiency, $E_{d}$, of primary drainage for packings of equal spheres with values obtained for sandstone and limestone (average for Edwards GC, Blocks 1 and 2) based on air/brine capillary pressure data.

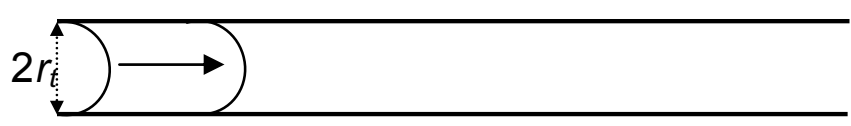

(i) reversible displacement in a cylindrical pore $(\xi=1)$

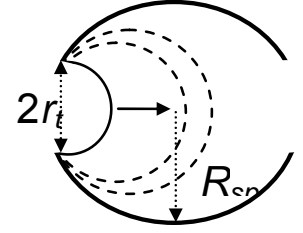

(ii) unstable displacement in a spherical cavity $(\xi=2.4)$

(a)

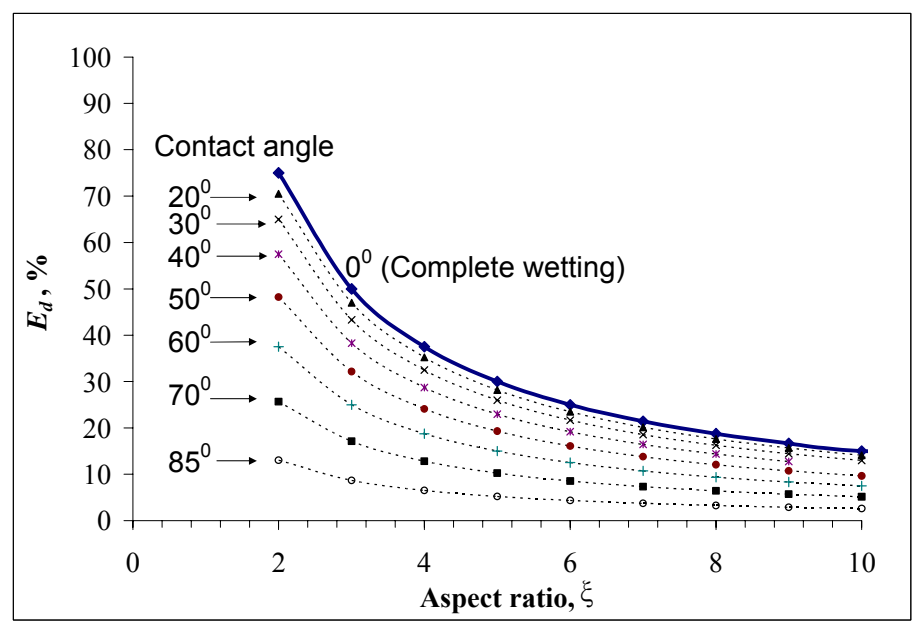

(b)

Fig. 6-12. Effect of aspect ratio on the efficiency of displacement, $E_{d,}$ for a spherical pore. (a) Cylindrical and spherical pores of equal volume. (b) $E_{d}$ vs $\xi$ (entry pressure of cavity assumed to be independent of contact angle). 
Task 7. Novel imbibition measurements on mixed-wet rock and network models.

Abstract

Counter-Current Spontaneous Imbibition (COUCSI) in porous media is driven by capillary forces. These forces generate an effective capillary pressure at an imbibition front but also oppose production of non-wetting phase at the outlet face. It is the difference in these pressures that draws in the wetting phase and pushes out the non-wetting phase. The effective capillary pressure at the wetting phase front under dynamic conditions is the ultimate driver of the imbibition process. Its value is crucial to mathematical simulation of spontaneous imbibition. A novel technique of measuring the effective capillary pressure at an imbibition front has been applied to seven sandstone cores. The core surface was sealed apart from one end face. The open end face of a sandstone core was butted to a short section of a finer pored rock referred to as the low permeability segment. A connection to a pressure transducer and an outlet for production of non-wetting phase was set in the other end face (referred to as the sealed end) of the core. Imbibition in some cases was first started as Cocurrent Spontaneous Imbibition (COCSI) with production of non-wetting phase through the sealed end face. The outlet through the sealed end face was then closed, and the increase in pressure was monitored as fluid redistributed within the core by capillary dispersion. Even without an initial period of co-current imbibition, the pressure rises to a maximum or approaches an asymptote as the imbibition front disperses. The maximum pressure is interpreted as the maximum effective capillary pressure at an imbibition front for COUCSI. In four of the sandstones, brine was the wetting phase and refined oil was displaced. In the remaining three, oil or brine was the wetting phase and air was displaced. Advance of the wetting fluid by capillary dispersion was detected by a series of electrodes for oil/brine and visually for air/oil and air/brine displacements. The maximum effective capillary pressure generated by the invading fluids ranged from $6.6 \mathrm{kPa}$ to $42 \mathrm{kPa}$ for Berea sandstone cores with permeabilities between $1.050 \mathrm{\mu m}^{2}$ and $0.06 \mathrm{\mu m}^{2}$. When air was the non-wetting phase, compression was a key factor in design and operation of the experiments. An estimate of the pore size below which gas is not produced by imbibition of liquid is provided.

\section{Introduction}

In Counter-Current Spontaneous Imbibition (COUCSI), a wetting phase front spontaneously invades a porous rock and forces the non-wetting phase to flow counter to the invading wetting phase. This process is important in the production of oil from fractured reservoirs. The imbibition of the Wetting Phase (WP) is in the direction of the negative capillary pressure gradient. The capillary pressure is highest at the WP front regardless of the shape of the saturation profile behind it. The lowest capillary pressure is at the open core face; this pressure, where Non-Wetting Phase (NWP) bubbles from the core, is referred to as the capillary back pressure, $P_{c b}$. The capillary back pressure at the exit face may oscillate locally at production sites but cannot be zero if the non-wetting phase is produced as bubbles (Li et al., 2004).

For one dimensional linear COUCSI many measurements of recovery versus time show linear dependence on the square root of time over a substantial period of the process. This form of dependence arises from the rate of advance depending only on the reciprocal of the distance advanced and implies that there is a period over which an effective driving capillary force is constant. The velocity of advance decreases significantly with time; thus the effective capillary pressure at the front cannot depend much on the interface velocity. Further confirmation that the effective capillary pressure is largely independent of velocity was provided by imbibition 
experiments with liquids of high viscosity. Results for conditions that gave very large decrease in rates of spontaneous imbibition still exhibited linear dependence on the square root of time (Fischer and Morrow, 2005).

Consider linear invasion of a wetting phase into a core with only one end face open. By definition, the constant driving force which can be identified with a constant effective capillary pressure at the WP front, $P_{c f}$, where

$$
P_{c f}=P_{n w, f}-P_{w, f}
$$

$P_{n w, f}$ is the pressure in the NWP and $P_{w, f}$ is the pressure in the WP. Small pores at the front can have higher capillary pressures than $P_{c f}$ but they do not determine the effective pressure directly because of the high local viscous dissipation and cross flow associated with their filling.

For a core initially saturated with NWP, there is only NWP between the front and the sealed end. Because there is no flow in the dead end pore space,

$$
P_{\text {end }}=P_{n w, f}
$$

Combining Eqs. 7-1 and 7-2 yields

$$
P_{\text {end }}=P_{c f}+P_{w, f}
$$

When the front is in movement, $P_{w, f}$ must be less than 0 (the pressure value in the WP reservoir) and so

$$
P_{\text {end }}<P_{c f}
$$

If there is no overall change in saturation and local redistribution of liquid is very slow, $P_{w, f}$ is 0 (the pressure value in the WP reservoir), and the pressure in all NWP contiguous to the dead end must be equal to $P_{\text {end }}$. Under this hypothetical condition

$$
P_{\text {end }}=P_{c f}
$$

Experiments have been designed so that production of non-wetting phase from the open end of the core is either prevented or severely limited. If the imbibition is begun as a COCSI process, the redistribution of fluids within the core corresponds to transition to what will be referred to as restricted COUCSI. The change in the dead end pressure was monitored during dispersal of the imbibition front.

\section{Experiments}

\section{General}

The technique used to measure the maximum effective capillary pressure at the front for COUCSI in Berea sandstone cores is as follows. A short segment of low permeability core was 
chosen which had significantly smaller pores than the main core. This acts as a semi-permeable membrane that potentially allows WP to enter the core and, ideally, prevents NWP from leaving. The external surface of the composite core (the short section plus the main core) was sealed with epoxy resin with the exception of the outer (open) face of the short core. A pressure tap for connection to a pressure transducer was set into the sealed end of the composite core. The core was then immersed in the wetting phase. Features of individual tests allowed invasion to some extent of wetting phase into the main core segment. For example, in two experiments, a vent on the pressure tap was left open to allow a short period of COCSI. After closing the vent, the rise in $P_{\text {end }}$ that accompanied transition from COCSI to restricted COUCSI was monitored while the fluids redistributed within the main core segment. The experiments were designed so that the development of the saturation profile given by transition to restricted COUCSI was essentially complete, as indicated by a maximum in $P_{\text {end }}$, before the front of the continuously dispersing wetting phase reached the dead end of the main core.

\section{Rock and fluid properties}

Eight Berea sandstones, of $3.8 \mathrm{~cm}$ diameter and about $7 \mathrm{~cm}$ length, with permeabilities ranging from $0.06 \mu \mathrm{m}^{2}$ to $1.067 \mu^{2}$ (see Table 7-1) were tested for brine/oil, oil/air, or brine/air imbibition. Refined oil (Soltrol 220, viscosity 0.0038 Pa.s) served as the oil phase. The brine contained 10,000 ppm NaCl (viscosity 0.00102 Pa.s). The air viscosity was 0.000018 Pa.s. The interfacial tension was $48.8 \mathrm{mN} / \mathrm{m}$ between brine and oil and $25.9 \mathrm{mN} / \mathrm{m}$ between oil and air.

\section{Measurements on composite cores}

Either a limestone $\left(0.003 \mu \mathrm{m}^{2}\right.$ permeability with a breakthrough pressure of $\left.163 \mathrm{kPa}\right)$ or a low permeability sandstone $\left(0.065 \mu \mathrm{m}^{2}\right)$ segment was butted to each tested sandstone core, with a 0.1 $\mathrm{cm}$ thick layer of powder derived from crushed Berea sandstone at the joint to ensure hydraulic contact. All surfaces of the composite core were sealed with transparent epoxy resin, except for the outer face of the low permeability segment. The general set-up of the apparatus is shown in Fig. 7-1. The change in pressure at the end of the core was monitored by with a pressure transducer. Saturation changes were determined gravimetrically. The volumetric fraction of pore space invaded by brine, $Q_{w} / V_{\phi}$ was calculated from weight change. When oil was the nonwetting phase, the distance of advance of brine from the open core face, $x_{f}$, was detected from onset of electrical conductivity between electrodes embedded in the core and an electrode in the brine.

When air was the non-wetting phase, the distance of advance of either oil or brine with time was monitored visually. A nylon tube with an inner diameter of $1.5 \mathrm{~mm}$ and an outer diameter of 3 $\mathrm{mm}$ was embedded in the core at the otherwise epoxy sealed end of the main segment. All experiments were conducted at room temperature (about $21^{\circ} \mathrm{C}$ ) and atmospheric pressure (79 $\mathrm{kPa}$ ). Results sometimes showed obvious, but minor, sensitivity to diurnal variations in temperature. Some details of experimental procedure varied with individual core tests and are given with the results. 


\section{Results}

\section{WATER-OIL}

\section{Test OW1}

Details of the composite core are given in Table 7-1. Both sections of the composite core were initially $100 \%$ saturated with oil. In this test, a period of COCSI was allowed with oil being produced from the end tube. Response of an electrode at the joint indicated that the COCSI front had crossed the joint after 0.45 hour. After 2.6 hours the front had penetrated $2.75 \mathrm{~cm}$ into the high $K$ core segment. The end tube was then closed to the atmosphere so that subsequent imbibition changed to COUCSI. At the start of the COUCSI, the end pressure rose quickly and reached its highest value, $12.17 \mathrm{kPa}, 0.37$ day after the start of COUCSI (see Fig. 7-2). Eventually, oil production was observed at the open face of the low $K$ segment and the measured end pressure started to fall. Without this production, the measured pressure would probably have reached a higher value. When the end tube was opened to the atmosphere, the end pressure fell to zero. Transition form COUCSI to COCSI resulted in oil recovery increase from $28 \%$ to $48 \%$ within 10 hours (see Fig. 7-2).

\section{Test OW2}

In this test, the low $K$ segment was initially saturated with brine and was then butted against an oil-saturated main segment. The composite core was then immersed in brine to initiate COUCSI imbibition. The end pressure rose to $11 \mathrm{kPa}$ within $2 \mathrm{hrs}$ and reached its highest value of 12.4 $k P a$ after 2.08 days. By that time, the front had penetrated $3.25 \mathrm{~cm}$ into the high $K$ core segment. The pressure was monitored for a further 1.8 days to confirm that end pressure was stable. There was no leakage of oil from the open face (unlike Test OW1 in which the low permeability oil segment was initially oil saturated). Movement of the water along the core was detected by the onset of electrical conductivity when the brine front contacted a particular electrode. The water invasion indicated in Fig. 7-3 by the change in oil content, $Q_{o} / V_{f}$, is ascribed to displacement of oil accompanied by compression of gas contained in the tube connected to the pressure transducer.

After 3.9 days, the core was sawed in half along its axis. Sharp increase in resistivity when an electrode contact was advanced along the exposed axis of the sliced core showed that the brine had penetrated $3.13 \mathrm{~cm}\left(x_{f} / L_{c}=0.45\right)$ into the core after 3.9 days.

\section{Test OW3}

For a sandstone of $0.492 \mu \mathrm{m}^{2}$ permeability ( see Table 7-1), the end pressure rose to $29.3 \mathrm{kPa}$ after 1.5 days and took about 8 days to reach to its highest value of $30 \mathrm{kPa}$ (see Fig. 7-4). (There was no oil production from the open face.) After 8 days, the electrical conductivity measurement indicated the brine had penetrated at least $3.6 \mathrm{~cm}$ into the main segment $\left(x_{f} / L_{c}=0.6\right)$. The maximum increase in average fractional brine saturation $Q_{w} / V_{\phi}$ of the main segment was 0.064 . This corresponded to the estimated volumetric compression of air in the tube connected to the transducer. As the gas in the tube becomes compressed, the measured end pressure became increasingly sensitive to variations in ambient pressure and temperature. The overall tendency for decrease in $\mathrm{P}_{\text {end }}$ after about 7 days is ascribed to slight change in core properties with length. After 12 days the end tube was opened and imbibition changed from COUCSI to COCSI; oil recovery increased from $6.4 \%$ to $52.4 \%$ within 1 day. 


\section{Test OW4}

The main sandstone segment was $6.175 \mathrm{~cm}$ long with permeability of $0.06 \mu \mathrm{m}^{2}$. This was the same sandstone that had served as the low permeability segment in Tests OW1 and OW2. The low permeability segment, cored from Texas Leuders limestone, was $1.489 \mathrm{~cm}$ long, and was initially saturated with brine.

With the low permeability sandstone as the main segment, the end pressure rose very quickly to over $40 \mathrm{kPa}$, within 1.2 days and increased only slightly over the next 7 days to $42 \mathrm{kPa}$. Electrical conductivity indicated that the front had penetrated at least $2.6 \mathrm{~cm}$ into the main segment $\left(x_{f} / L_{c}=0.42\right)$. No oil production was observed at the open face throughout the 8 days test. The increase in invaded brine saturation by compression of gas in the tube to the transducer was $5.1 \%$.

\section{AIR-OIL OR AIR-WATER}

\section{Test AW1}

With a gas as the non-wetting phase, compressibility effects become highly significant. A preliminary test of restricted imbibition of brine with air as the non-wetting phase was made with a $65 \mathrm{md}$ dry Berea sandstone as the main segment and a $3 \mathrm{md}$ brine-saturated limestone as the semi-permeable membrane (Fig. 7-6). The air breakthrough pressure for the limestone was 163 $\mathrm{kPa}$. From direct observation, the interface had advanced to the end of the core within 0.46 days. However the end pressure was still rising. The end pressure attained a steady value of $26 \mathrm{kPa}$ whereas the end pressure predicted by scaling interfacial curvatures determined by the oil/brine results was about twice this value. In tests with gas as the non-wetting phase, even though a semi-permeable membrane at the open end of the composite core prevents production of gas, compression of gas within the core allows imbibition of the wetting phase. For the results shown in Fig. 7-6, the compression of gas within the main core segment is not sufficient to stop the front from reaching the end of the core before the recorded end pressure attains the expected $P_{c f}$ value for restricted COUCSI.

\section{Test AO1}

Use of oil as the invading phase (WP) and air as the displaced phase (NWP) was tested next. As for AW1, the main segment was initially dry $65 \mathrm{md}$ Berea sandstone which was sealed with epoxy resin except at one end face. An oil saturated paste of finely crushed Berea sandstone was spread between the face and an oil saturated $3 \mathrm{md}$ limestone end piece that was sealed at the side to have two ends open. The periphery of the joint between the low permeability end piece and the main sample was then sealed.

After submerging the core in oil, the gas pressure in the core was elevated to $13.5 \mathrm{kPa}$ by air injection via the sealed end in order to reduce the dominant effect of compression observed for Test A1. The outlet end of the core was then closed. The end pressure continued to rise slowly above the initial value of $13.5 \mathrm{kPA}$ (guage) and reached $21.3 \mathrm{kPa}$ after 8.8 days. Monitoring of the pressure for a further 1 day confirmed that a stable end pressure had been attained. During uptake of brine through the open end of the composite core, there was no air production at the open face. The increase in brine saturation was determined from the change in volume with gas pressure with the assumption that dissolution of air could be neglected. The detected distance of 
advance of the imbibition front after 9.9 days was 0.64 of the main core length. The outlet tube was then opened and an additional $0.48 \mathrm{PV}$ of water rapidly advanced to the end of the main core segment by unrestricted COCSI (see Fig. 7-7).

\section{Test AO2}

The experiment on Core AO2, was first deliberately run in COCSI mode for 12 minutes by leaving the end tube open to atmosphere. This allowed the imbibition front to advance into the main core to about 0.09 of $L_{c}$. The pressure of the air in the core was then raised to $6.5 \mathrm{kPa}$ by injection. As, imbibition progressed, the end pressure rose slowly and reached a plateau at 14.5 $\mathrm{kPa}$ after 18 days and showed no further change over the next 4 days. $Q_{w} / V_{\phi}$ and $x_{f} / L_{c}$ also reached stable values of $0.112 \mathrm{PV}$ and $0.43 L_{c}$ respectively. Finally, COUCSI was terminated by opening the outlet tube. Oil advanced rapidly by COUCSI to the end of the core and the brine content of the core increased from $0.112 \mathrm{PV}$ to $0.637 \mathrm{PV}$.

\section{Test AO3}

Composite core AO3 was formed from a $7.928 \mathrm{~cm}$ main segment of dry $1042 \mathrm{md}$ Berea sandstone butted against a $1.884 \mathrm{~cm}$ long $65 \mathrm{md}$ segment of Berea sandstone. A layer of paste of ground Berea sandstone of $0.112 \mathrm{~mm}$ thickness was applied at the joint and the periphery was sealed with epoxy resin. The core was then dried. Oil was allowed to invade the composite core by COCSI. After $42 \mathrm{~min}$ the front was observed to have reached the joint. After 4.5 hours, the front had advanced to $0.45 L_{c}$ of the long core segment and the average oil saturation in the core $\left(Q_{w} / V_{\phi}\right)$ was $20.6 \%$. The outlet valve was then closed and the end pressure rose from 0 to $6.6 \mathrm{kPa}$ within 2.8 days. The end pressure $P_{\text {end }}$, the influx $Q_{w}$, and the observed distance of advance, $x_{f}$, remained constant for 1 day. The outlet valve was then opened and the end pressure fell to zero. The water influx and distance of advance increased sharply. An additional $0.354 \mathrm{PV}$ of gas was produced. The results are shown in Fig. 7-9. No gas production was observed from the open end of the core throughout this test.

\section{Tests AW4 and AO4}

A comparison was next made between air/oil and air/water imbibition for the same core segment. Imbibition of water was tested first, starting with composite core AW4 initially dry. The air pressure in the core was raised to $29.8 \mathrm{kPa}$ by air injection prior to COUCSI. After 1 day $P_{\text {end }}$ increased to $31.5 \mathrm{kPa}$ because of restricted COUCSI. The air pressure in the core was then raised to $38.0 \mathrm{kPa}$ so that predicted value of $P_{\text {end }}$ was exceeded. After 1 day $P_{\text {end }}$ decreased to $36.8 \mathrm{kPa}$. The extrapolated value of stable $P_{\text {end }}\left(=P_{c f}\right)$ from the rising and falling pressure data is about $34.7 \mathrm{kPa}$.

After the air/brine test, the two segments were separated. The main segment was dried and reused in making air/oil imbibition measurements on core AO4. The air pressure in the core was raised to $12.1 \mathrm{kPa}$ prior to COUCSI. The measured $P_{\text {end }}$ tended to increase with time but showed significant fluctuation around $12.3 \mathrm{kPa}$. After scaling for interfacial tension, the extrapolated interfacial curvature $\left(P_{c f} / \sigma\right)$ for water/air imbibition of about $0.475 \mu^{-1}$ is close to the average value indicated by the fluctuating value of $P_{\text {end }}\left(0.475 \mu \mathrm{m}^{-1}\right)$ for air/oil imbibition. 


\section{Results and Discussion}

\section{Maximum capillary pressure at the imbibition front.}

The maximum stable values of $\mathrm{P}_{\text {end }}$ before the front reaches the end of the core provide an estimate of the effective capillary pressure acting at the imbibition front. The maximum values of $\mathrm{P}_{\text {end }}$ for Berea sandstones with permeabilities between 1.067 to $0.06 \mu^{2}$ ranged from 6.6 to 21.3 $\mathrm{kPa}$ for water/oil. For oil/air, the maximum values of $\mathrm{P}_{\text {end }}$ ranged from 12.4 to $42 \mathrm{kPa}$. For sandstones of the same permeability the effective frontal curvatures $\left(P_{c f} \sigma\right)$ are all close to equal for water/oil and oil/air cases (see Table 7-1).

Frontal capillary pressures in low permeability Berea sandstone determined by simulation have been reported ( $\mathrm{Li}$ et al., 2004). The simulation was based on the assumption that the relative permeabilities are identical and capillary pressures are scaled by the Leverett (1941) function for any Berea sandstone. The simulations predict values of $P_{c f}$ that are about double the maximum pressures measured in the present work. However, simulation of dynamic spontaneous imbibition using conventional relative permeability and capillary pressure relationships is problematic because of the physical nature of the imbibition process including the sharpness of the imbibition front (Fischer et al. 2006).

\section{Dispersion during restricted COUCSI}

The transition to restricted COUCSI results in dispersion of the front because of capillary forces. As the disperse region grows, the viscous pressure gradients in the vicinity of the front decrease with time. A remarkable feature of the results is that in many instances $\mathrm{P}_{\text {end }}$ remains essentially constant as the front disperses.

The simplest explanation of the dispersion of the wetting front is provided by consideration of the behavior of independent parallel tubes having a range of diameters. During co-current imbibition, the interface in the largest diameter tube lead and the interfaces in the smallest diameter tubes lag. If the tubes have perfect cross-flow between them, imbibition behavior is more akin to that observed for sandstone. The interfaces group together into a front in which the small tubes have menisci that lead the menisci in the larger tubes. In effect the small tubes are fed from the flow in the large tubes. If all of the tubes with cross flow are suddenly capped, the menisci in the small tubes continue to advance, but the menisci in the large tubes moves backwards and may even reach the tube entrance. As the velocity in the small tubes decreases, the measured pressure continues to build and the 'front' continues to disperse as more large pores empty. Imbibition eventually ceases once the wetting liquid occupies the full length of a group of the smaller tubes. Just how many of the smaller tubes fill depends on the amount of wetting phase in the larger tubes at the time the tubes are capped.

Naturally occurring porous media such as rocks cannot be modeled satisfactorily as a bundle of tubes, do not have perfect cross-flow, and the pores do not have uniform cross section. They are better regarded as an interconnected network of cavities and constrictions. For a small pore to exert a capillary driving pressure consistent with its size, it needs to be connected to the continuous wetting phase front ( $\mathrm{Li}$ et al., 1986). The overall effect of this percolation connectivity requirement is to severely restrict the distance of advance of the wetting phase through the smaller pores. Consequently the wetting phase does not necessarily reach the end of 
the sample during redistribution of the front. However, percolation theory has mainly been developed for quasi-static displacements rather than dynamic. It is a remarkable experimental fact that once the measured end pressure has built up to its maximum value, it does not vary greatly during the course of increased dispersion of the front (see Figs 7-3 through 7-9) When the flow is stopped, fluid re-distributes at the front and approaches a static condition related to the dynamic saturation profile from which it was formed.

\section{Average wetting phase saturation behind the dispersed front}

For oil/air imbibition, the average wetting phase saturation behind the dispersed front can be estimated from the position of the directly visible front and the amount of oil imbibed (from the weight change). For example, for Core M1A the saturation at the front can be estimated by the quotient of the final $Q_{\mathrm{w}} / V_{\phi}$ over the final $x_{\mathrm{f}} / L_{\mathrm{c}}$. The final $Q_{\mathrm{w}} / V_{\phi}$ was 0.112 and the corresponding $x_{\mathrm{f}} / L_{\mathrm{c}}$ was 0.43 . Consequently the average saturation behind the front, $S_{\mathrm{wf}}$, is 0.26 . This is the same as the value estimated previously by simulation $\left(S_{\mathrm{wf}}=0.26\right)$ for Berea Sandstone with permeability of $0.503 \mu^{2}$ (M3A) (Li et al., 2004).

Another example is the measurement of $P_{c f}$ in Core M1A. Because the final $Q_{\mathrm{w}} / V_{\phi}$ was 0.177 and the corresponding $x_{\mathrm{f}} / \mathrm{L}_{\mathrm{c}}$ was 0.64 , the saturation behind the front, $S_{\mathrm{wf}}$, was 0.277 . Although lower than the value estimated by simulation $\left(S_{\mathrm{wf}}=0.345\right)$ for $.070 \mu \mathrm{m}^{2}$ permeability Berea sandstone in the oil/air case, the value is about the same as most of those estimated by simulation ( 0.26 to 0.32) (Li et al., 2004).

\section{Compression effects}

The effect of compressibility when the non-wetting phase is a gas raises an interesting point. Consider the advance of wetting phase into a $100 \%$ gas saturated core at atmospheric pressure. As the wetting phase front advances, trapped non-wetting phase is left behind at essentially the same pressure as the NWP at the front. In the reported liquid/gas experiments, increase in gas pressure to a maximum is followed by gas production. However, if the volume of the entrapped gas is about half of the total pore space and the effective gauge pressure at the advancing front is about equal to atmospheric pressure, from the ideal gas law, it is clear that the volume of trapped air can account for all of the air initially present in the core. The phenomenon of imbibition without gas production was reported by Iounnou et al. (2003) for a ceramic with fine pores. As a rule of thumb, with water, pores with $1.5 \mu \mathrm{m}$ radius produce a capillary pressure of about 1 bar. So any air-filled porous material with residual saturation equal to about $50 \%$ of the pore space will only exhibit production of gas bubbles if its pore diameters are greater than about $3 \mu \mathrm{m}$.

\section{Imbibition into crude oil saturated (COS) and mixed wettability cores}

Work on COS and mixed-wettability cores is in progress and will be reported in detail in the next semi-annual report.

\section{Nomenclature}

$D \quad$ core diameter $(m)$

$K \quad$ permeability to air $\left(m^{2}\right)$

$L_{c} \quad$ core length $(m)$

$P_{c} \quad$ capillary pressure $(P a)$

$P_{c b} \quad$ capillary back pressure $(P a)$ 
$P_{c f} \quad$ capillary pressure at the imbibition front $(\mathrm{Pa})$

$P_{\text {end }} \quad$ pressure measured at the dead end $(\mathrm{Pa})$

$P_{n w, f} \quad$ pressure in non-wetting phase at the front $(\mathrm{Pa})$

$P_{w, f} \quad$ pressure in wetting phase at the front $(\mathrm{Pa})$

$Q_{w} \quad$ cumulative input of wetting phase $\left(\mathrm{m}^{3}\right)$

$\left(Q_{w} / V_{\phi}\right)_{\text {COCSI }}$ the cumulative fractional imbibition volume at the end of COCSI

$\left(Q_{w} / V_{\phi}\right)_{\text {COUCSI }}$ the cumulative fractional imbibition volume at the end of COUCSI

$S_{w f} \quad$ frontal saturation

$x_{f} \quad$ distance of the front from the joint $(m)$

$V_{\phi} \quad$ pore volume $\left(\mathrm{m}^{3}\right)$

$\sigma \quad$ interfacial tension $(\mathrm{N} / \mathrm{m})$

$\phi \quad$ porosity

\section{Acronyms/abbreviations}

WP wetting phase

NWP non-wetting phase

COCSI co-current spontaneous imbibition

COUCSI counter-current spontaneous imbibition

\section{References}

Fischer, H. and Morrow, N.R., "Scaling of oil recovery by spontaneous imbibition for matched viscosities" SPE96812, presented at the SPE Annual Technical Conference, Dallas, TX, Oct. 9$12,2005$.

Fischer, H., Wo, S, and Morrow, N.R., "Modeling the Effect of Viscosity Ratio on Spontaneous Imbibition" SPE 102641 to be presented at the 2006 SPE ATCE, San Antonio, TX, Sept. 24-27.

Iounnou I., Hall C., Wilson M. A, Hoff W. D and Carter M. A, 2003, Direct Measurement of the Wetting Front Capillary Pressure in a Clay Brick Ceramic, J. Phys. D: Appl. Phys. 36, 31763182 .

Leverett, M.C.: "Capillary Behavior in Porous Solids", Trans. AIME, 142, (1941), 152.

Li, Y., Laidlaw, W.C., and Wardlaw, N.C., 1986, Sensitivity of Drainage and Imbibition to Pore Structure as Revealed by Computer Simulation of Displacement Process, Advances in Colloid and Interface Science, 26, 1-68.

Li, Y., Ruth D., Mason G. and Morrow N.R., 2004, Pressure acting in counter-current spontaneous imbibition, Journal of Petroleum Science and Engineering 52, 2006, 87-99.

Wardlaw N. C. and Li Y., 1987, Pore-Throat Size Correlation from Capillary pressure curves, Transport in Porous Media, 2, 597-614. 
Table 7-1 Properties of Composite Core Segments

\begin{tabular}{|c|cccc|cccccc|}
\hline $\begin{array}{c}\text { TEST } \\
\text { ID }\end{array}$ & $\begin{array}{c}\mathrm{D} \\
(\mathrm{cm})\end{array}$ & $\begin{array}{c}L_{c} \\
(\mathrm{~cm})\end{array}$ & $\begin{array}{c}\mathrm{K} \\
\left(\mu \mathrm{m}^{2}\right)\end{array}$ & $\begin{array}{c}\phi \\
(\%)\end{array}$ & $\begin{array}{c}\mathrm{D} \\
(\mathrm{cm})\end{array}$ & $\begin{array}{c}L_{c} \\
(\mathrm{~cm})\end{array}$ & $\begin{array}{c}\mathrm{K} \\
\left(\mu \mathrm{m}^{2}\right)\end{array}$ & $\begin{array}{c}\phi \\
(\%)\end{array}$ & $\begin{array}{c}P_{c f} \\
(\mathrm{kPa})\end{array}$ & $\begin{array}{c}P_{c f} / \sigma \\
\left(\mu \mathrm{m}^{-1}\right)\end{array}$ \\
\hline \multicolumn{10}{|c|}{ WATER/OIL } \\
\hline OW1 & 3.789 & 2.069 & 0.065 & 17.1 & 3.794 & 6.88 & 1.052 & 22.1 & $>12.17$ & $>0.249$ \\
OW2 & 3.795 & 2.351 & 0.065 & 17.1 & 3.795 & 6.967 & 1.067 & 22.2 & 12.4 & 0.254 \\
OW3 & 3.8 & 1.714 & 0.003 & 16.8 & 3.796 & 6 & 0.492 & 20.2 & 30.0 & 0.614 \\
OW4 & 3.801 & 1.489 & 0.003 & 16.8 & 3.795 & 6.175 & 0.060 & 19.6 & 42.0 & 0.860 \\
\hline \multicolumn{10}{|c|}{ OIL/AIR or WATER/AIR } \\
\hline AW1 & 3.775 & 1.595 & 0.003 & 16.8 & 3.796 & 6.91 & 0.065 & 17.1 & $>26.0$ & $>0.356$ \\
AO1 & 3.822 & 1.67 & 0.003 & 16.9 & 3.795 & 6.175 & 0.060 & 16.9 & 21.3 & 0.823 \\
AO2 & 3.798 & 1.732 & 0.003 & 16.8 & 3.796 & 6 & 0.492 & 20.2 & 14.5 & 0.560 \\
AO3 & 3.8 & 1.88 & 0.065 & 17.1 & 3.79 & 7.928 & 1.042 & 22.1 & 6.6 & 0.255 \\
AO4 & 3.822 & 1.371 & 0.002 & 16.9 & 3.796 & 6.17 & 0.617 & 20.4 & 12.3 & $\sim 0.48$ \\
AW4 & 3.76 & 1.636 & 0.002 & 16.6 & 3.796 & 6.17 & 0.617 & 20.4 & 34.5 & $\sim 0.47$ \\
\hline
\end{tabular}

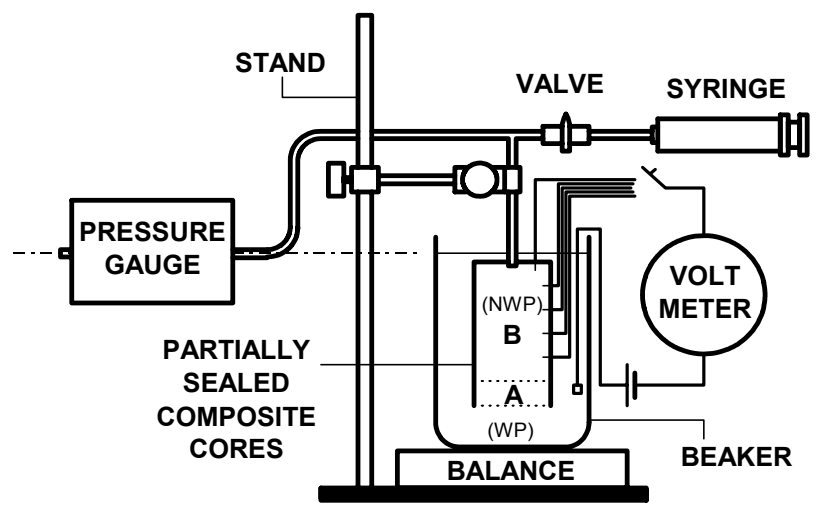

Fig. 7-1 Apparatus for measurement of the frontal capillary pressure in COUCSI. A is the low permeability core segment. $B$ is the high permeability core segment. 


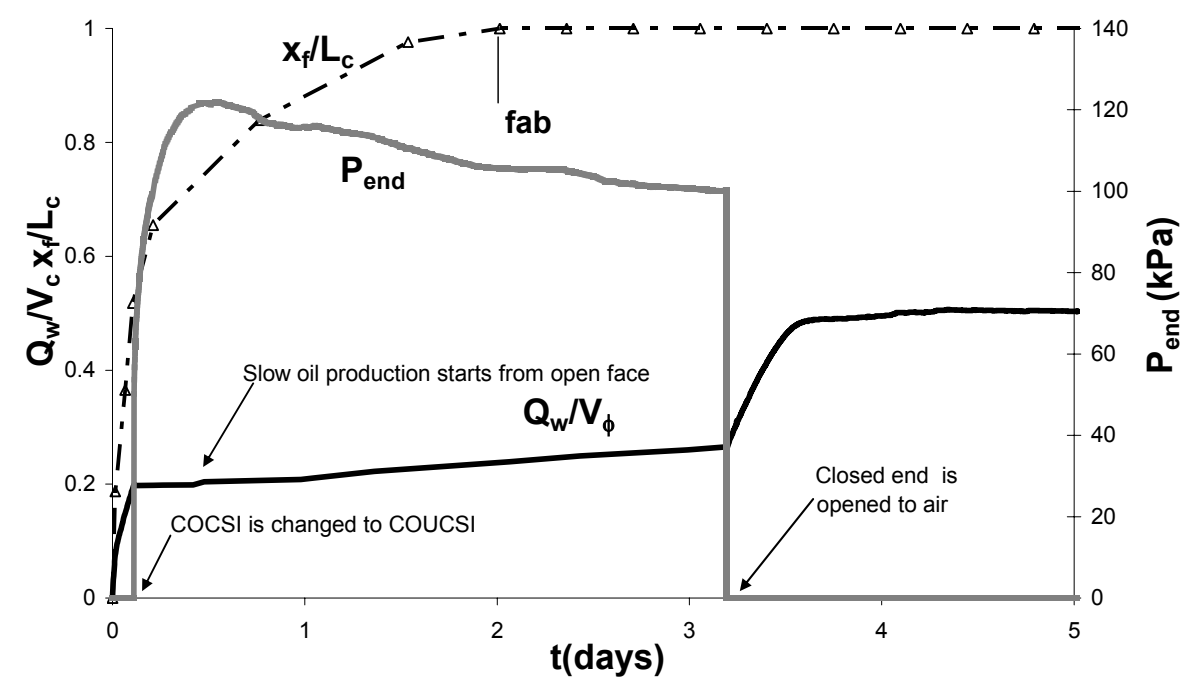

Fig. 7-2 Distance of advance of brine, average brine saturation and end pressure, versus time for Test OW1. Imbibition was changed from COUCSI to COCSI after 12 days by opening the end tube to atmosphere.

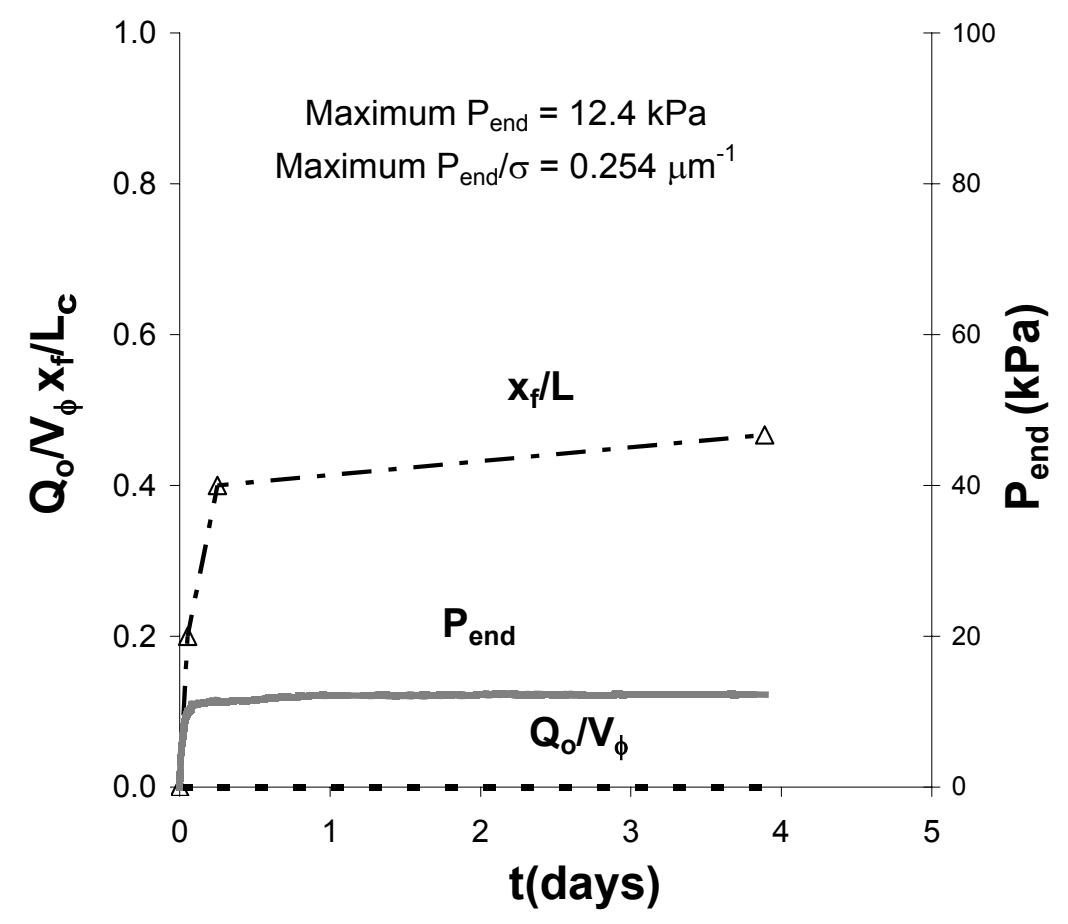

Fig. 7-3 Distance of advance of brine, average brine saturation and end pressure, versus time for Test OW2. 


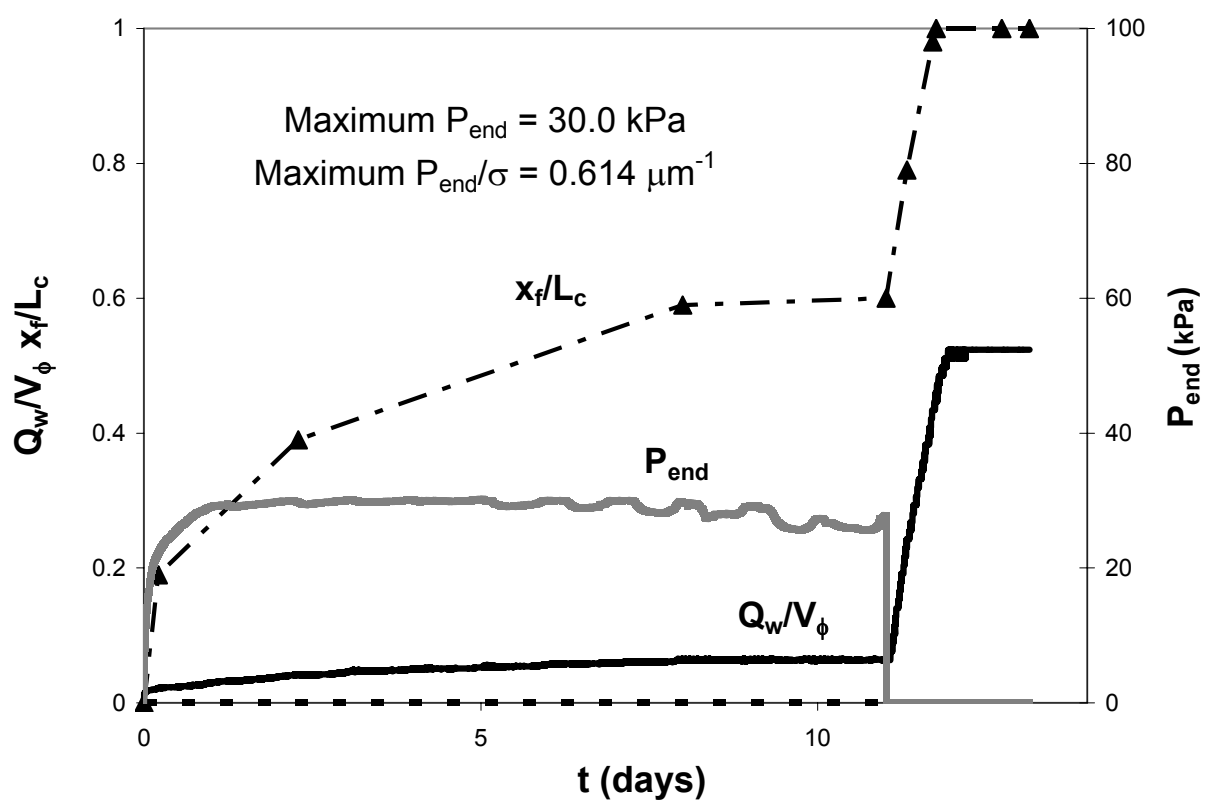

Fig. 7-4 Distance of advance of brine, average brine saturation, and end pressure versus time for Test OW3. Imbibition was changed from COUCSI to COCSI after 12 days by opening the end tube to atmosphere.

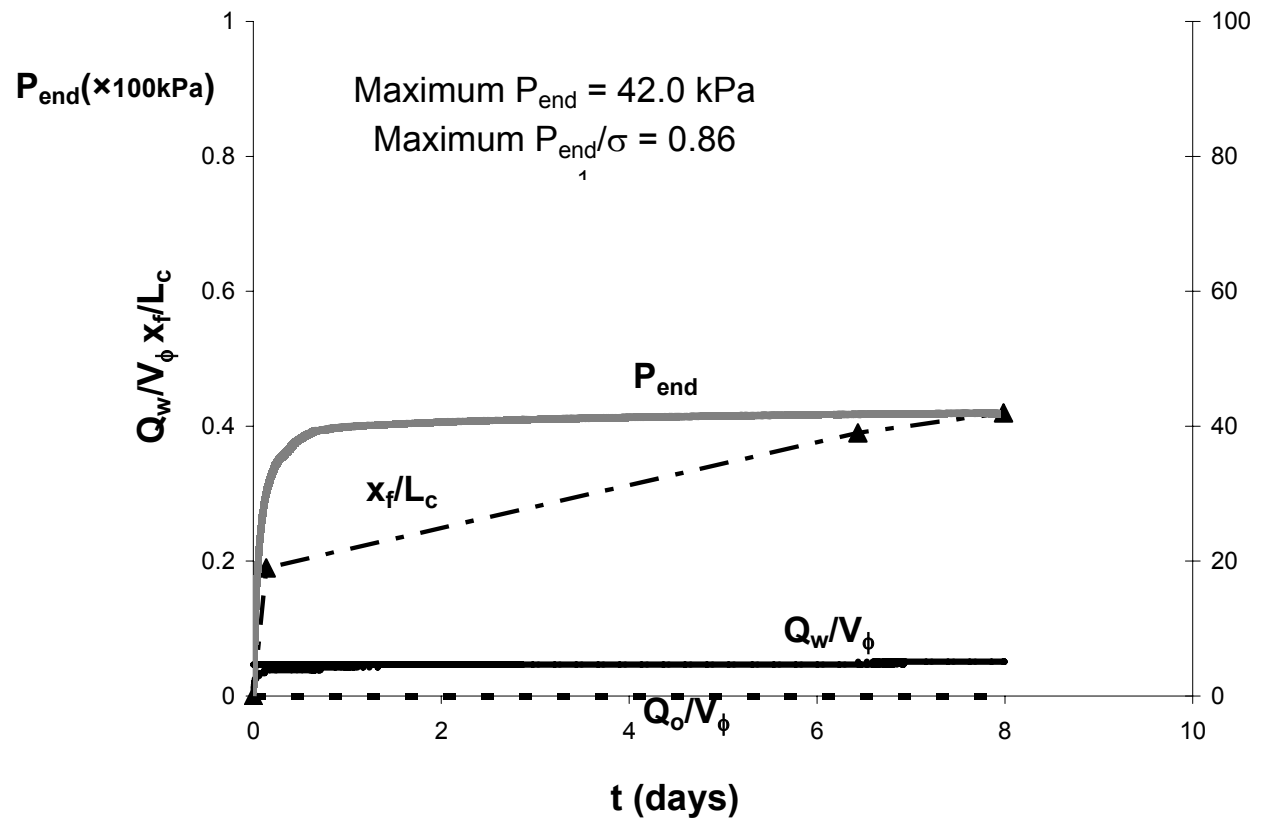

Fig. 7-5. Distance of advance of brine, average brine saturation and end pressure, versus time for Test OW4. 


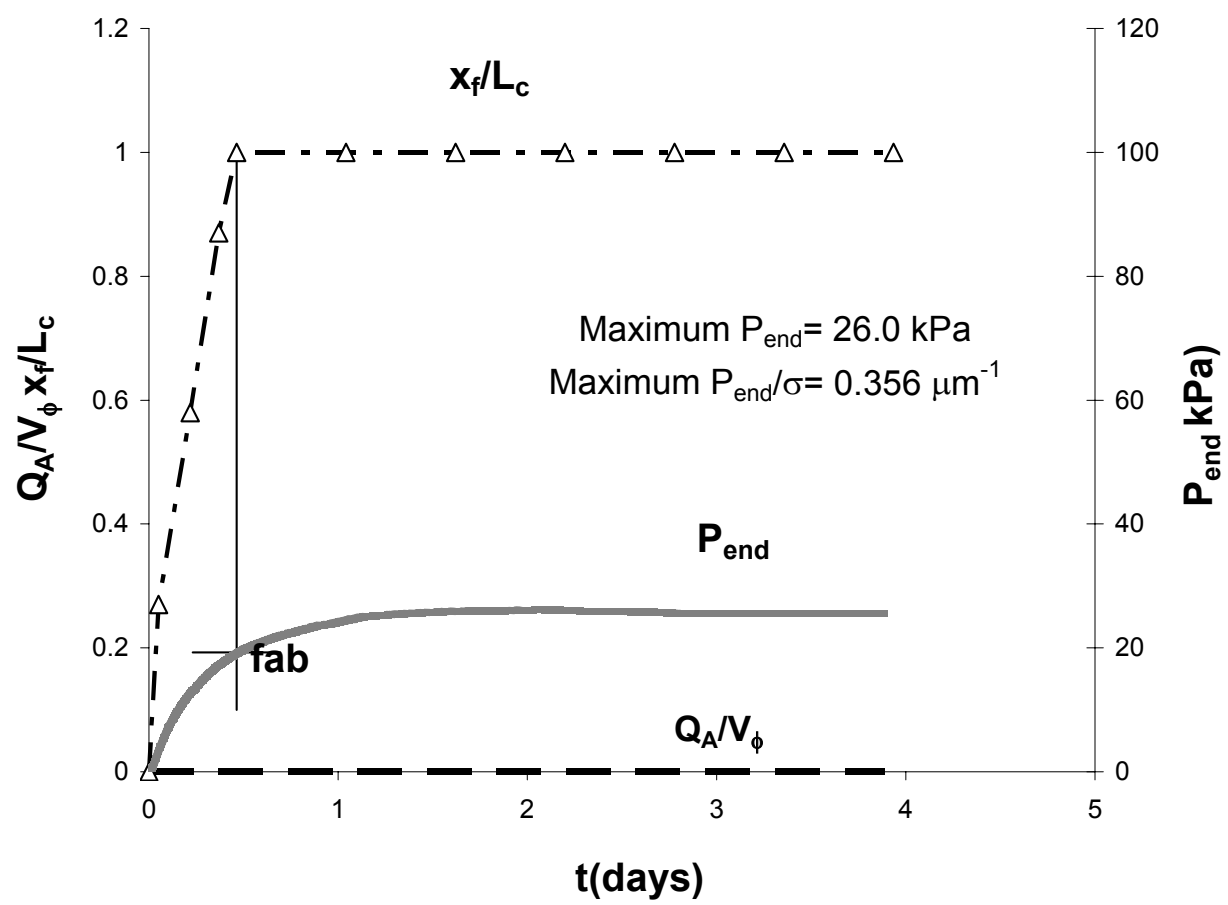

Fig. 7-6 Distance of advance of brine, average brine saturation and end pressure, versus time for Test AW1. Because of compression of the gas, the front reached the end of the core (fab) before $P_{\text {end }}$ attains the predicted value of $P_{c f}$.

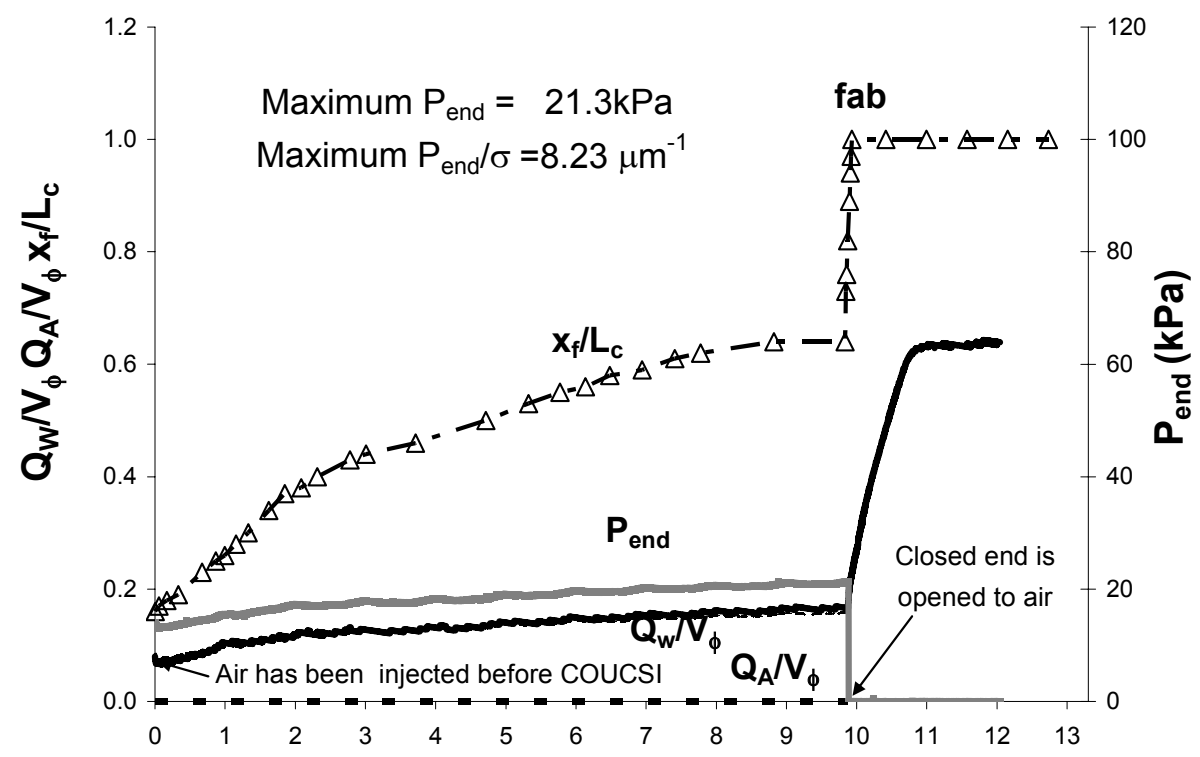

Fig. 7-7 Distance of advance of brine, average brine saturation, gas production and end pressure, versus time for Test A01. The initial gas pressure was raised to $13.5 \mathrm{kPA}$. 


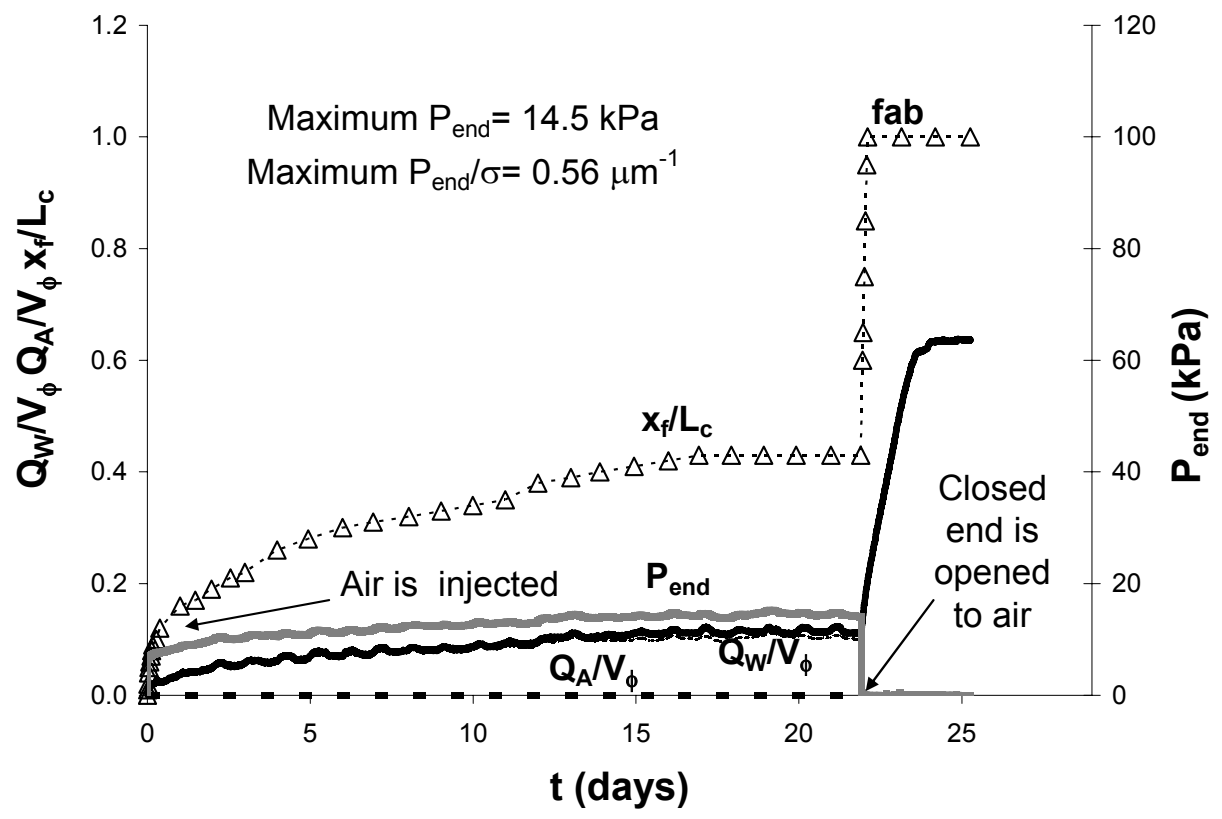

Fig. 7-8 Distance of advance of brine, average brine saturation, gas production and end pressure, versus time for Test AO2. After a short period of COCSI, the initial gas pressure was raised to 13.5 kPA.

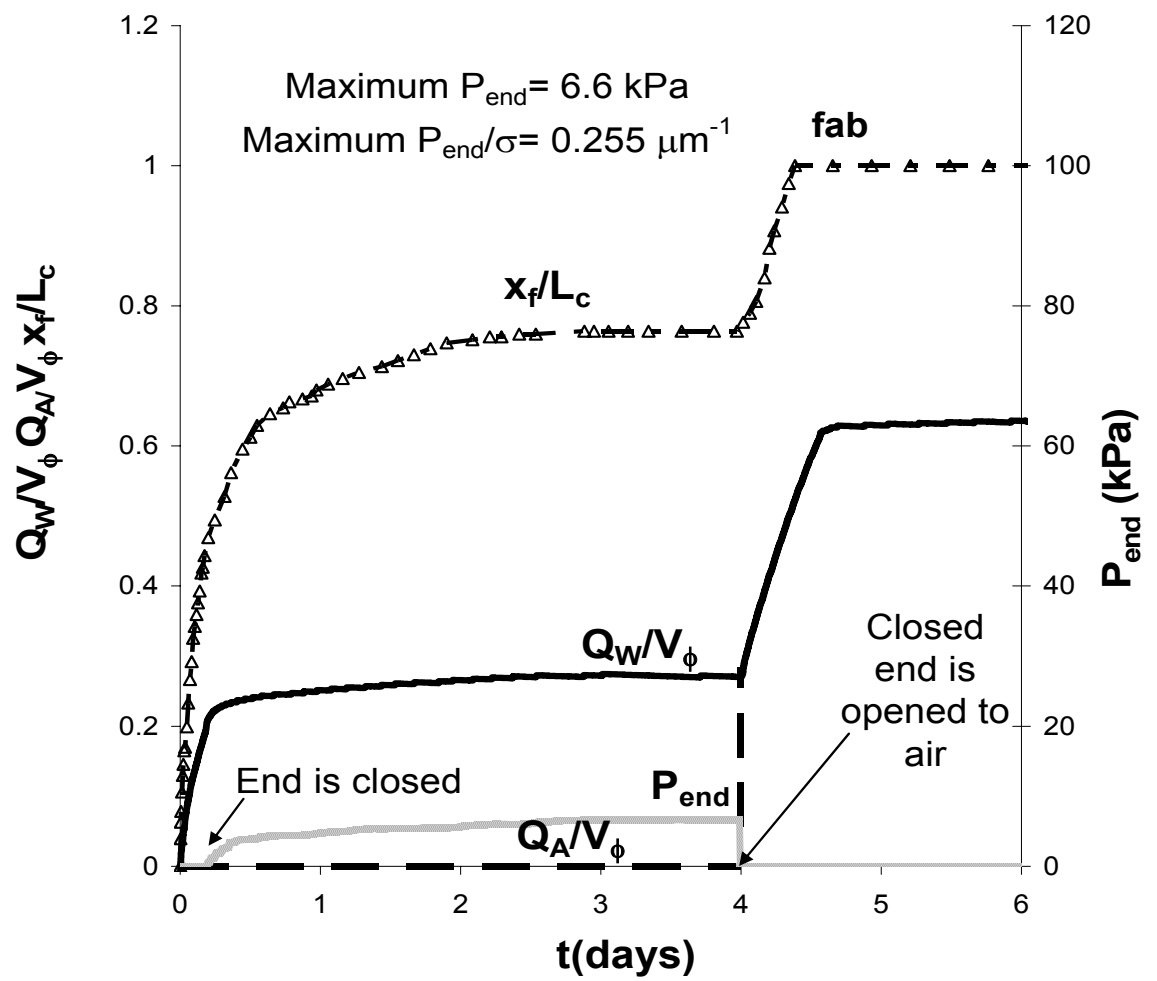

Fig. 7-9 Distance of advance of brine, average brine saturation, gas production and end pressure, versus time for Test AO3. After a short period of COCSI, the initial gas pressure was raised to $13.5 \mathrm{kPA}$. 


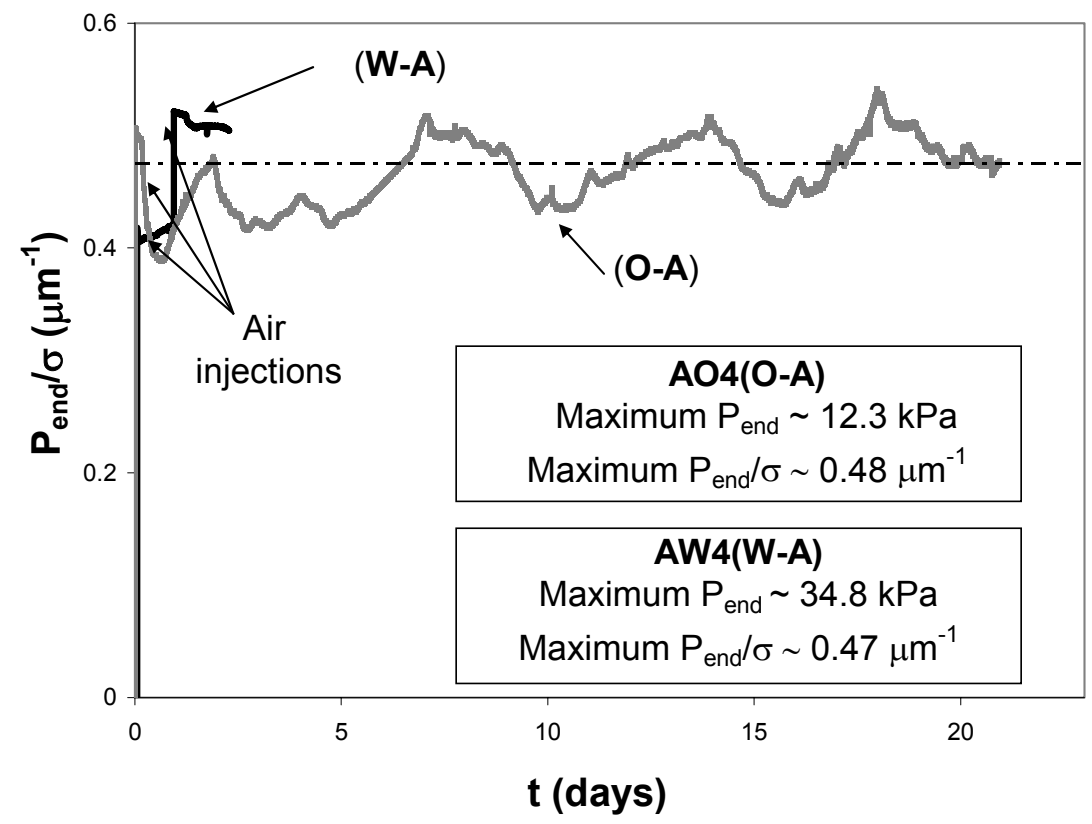

Fig. 7-10 Interfacial curvature versus time for air/brine and air/oil imbibition. Extrapolated values for conditions of both increasing and decreasing $P_{\text {end }}$ for air/brine are consistent with average values of $P_{\text {end }}$ for air/oil imbibition for composite cores AO4 and AW4.

Task 8. Application of network/numerical model to mixed wet rocks.

Work on simulation of imbibition by mixed-wet rocks is in progress.

Task 9. Increased oil recovery by spontaneous imbibition.

\section{Introduction}

Methods of improved recovery by enhanced imbibition either through use of surfactants or by manipulation of brine composition or some combination of both are being investigated. Austad and co-investigators (Strand et al., 2003) recently reported that the concentration of sodium sulphate had a major impact on oil recovery from chalk when cationic surfactants were used to promote spontaneous imbibition at $40^{\circ} \mathrm{C}$. The effect of surfactant and $\mathrm{Na}_{2} \mathrm{SO}_{4}$ on enhanced oil recovery through spontaneous imbibition is reported here for crude oil/brine/rock combinations from a dolomite reservoir.

\section{Experimental}

\section{$\underline{\text { Rocks }}$}

Rock samples are dolomite, taken from depth ranging from 4378 to $4392 \mathrm{ft}$, from San Andres formation of Fuhrman-Mascho Field of Permian Basin, Texas. The rock properties are listed in Table 9-1. 
Crude oil

San Andres formation crude oil has viscosity of $9.9 \mathrm{cP}$ at reservoir temperature, $40^{\circ} \mathrm{C}$, with density of $0.8920 \mathrm{~g} / \mathrm{cm}^{3}$ at $20^{\circ} \mathrm{C}$. The crude oil contains $1.0 \%$ asphaltenes. The interfacial tension between the crude oil and seawater is $20.3 \mathrm{mN} / \mathrm{m}$ at $40^{\circ} \mathrm{C}$.

$\underline{\text { Brines }}$

Two types of brine, simulated seawater and San Andres formation brine, are used in the tests, with the total dissolved solids (TDS) of 36.779 and $75.788 \mathrm{~g} / \mathrm{L}$, respectively (see Table 9-2).

$\underline{\text { Surfactants }}$

One nonionic surfactant, TOMADOL's 91-8- $\mathrm{RO}-\left(\mathrm{CH}_{2} \mathrm{CH}_{2} \mathrm{O}\right)_{8} \mathrm{H}, \mathrm{R}=\mathrm{C}_{9-11}$, was tested with concentration of $3000 \mathrm{ppm}$.

$\underline{\text { Imbibition }}$

In initial tests, dry rock samples were directly saturated with the crude oil to obtain baseline results for an initial water saturation of zero. The oil-saturated cores were then aged in sealed metal cells filled with the parent crude for ten days at $40^{\circ} \mathrm{C}$.

Oil recovery tests started with spontaneous imbibition of seawater at $40^{\circ} \mathrm{C}$. After oil recovery ceased, the samples were switched to surfactant solution. Oil recovery in percentage of the original oil in place was recorded against time.

\section{Results and discussion}

Oil recovery from spontaneous imbibition of brine

Results of oil recovery through spontaneous imbibition of seawater are plotted in Figs. 1a and $1 \mathrm{~b}$, and listed in Table 9-3 for the ten cores. There is a trend for lower permeability cores (less than $2 \mathrm{md}$ ) to have higher oil recovery (higher than 30\% OOIP) than higher permeability ones (greater than $40 \mathrm{md}$, less than 7\% OOIP ), except for Core $4384.9 \mathrm{~d}$ ( $82.4 \mathrm{md}, 18.8 \%$ OOIP) and $438.88 \mathrm{c}(1.6 \mathrm{md}, 6.2 \%$ OOIP). This is in line with the likely original mixed wettability condition of the reservoir whereby the lower permeability regions, as represented by core plugs, tend to be more water wet than the higher permeability regions.

Effect of T91-8 and $\mathrm{Na}_{2} \underline{\mathrm{SO}}_{4}$ on enhanced oil recovery through spontaneous imbibition At the termination of imbibition of seawater, the cores were transferred to surfactant solution prepared with simulated Fuhrman-Mascho formation brine with different concentrations of $\mathrm{Na}_{2} \mathrm{SO}_{4}$. The results are also plotted in Figs. 1a and $1 \mathrm{~b}$, and listed in Table 9-3. Overall, the nonionic surfactant T91-8 increases oil recovery from the dolomite rocks. Contrary to the results for imbibition of seawater, the higher permeability cores have higher incremental oil recovery (greater than 14\% OOIP) from the surfactant action than the lower permeability ones ( less than 10\% OOIP), except for Core $4379.4 \mathrm{c}$ ( $47.3 \mathrm{md}$, which did not respond to surfactant), and $4378.8 \mathrm{c}$ (1.6md, $18.6 \%$ OOIP for the surfactant action). In contrast to the response reported for chalk, incremental oil recovery did not change systematically with increase of $\mathrm{Na}_{2} \mathrm{SO}_{4}$ concentration from 0 to $6280 \mathrm{ppm}$ for the tested dolomite. 


\section{Reference}

Strand, S., Standnes, D.C., and Austad, T., " Spontaneous Imbibition of Aueous Surfact Solutions into Neutral to Oil-West Carbonate Cores: Effect of Brine Salinity and Composition" Energy \& Fuels, 2003, 17, 1133-1144.

Table 9-1 Rock properties

\begin{tabular}{|l|c|c|c|c|}
\hline Cores & $\mathrm{D}, \mathrm{cm}$ & $\mathrm{L}, \mathrm{cm}$ & $\mathrm{K}_{\mathrm{g}}, \mathrm{md}$ & $\phi, \%$ \\
\hline $4378.9 \mathrm{~d}$ & 3.784 & 4.483 & 144 & 0.1384 \\
\hline $4380 \mathrm{~b}$ & 2.511 & 5.880 & 131 & 0.1108 \\
\hline $4380 \mathrm{a}$ & 2.514 & 5.458 & 125 & 0.1061 \\
\hline $4384.9 \mathrm{~d}$ & 2.518 & 5.469 & 82.4 & 0.1598 \\
\hline $4379.4 \mathrm{c}$ & 2.512 & 4.803 & 47.3 & 0.0810 \\
\hline $4378.8 \mathrm{c}$ & 2.528 & 4.643 & 1.6 & 0.1384 \\
\hline $4384.6 \mathrm{~d}$ & 2.518 & 5.140 & 1.6 & 0.1308 \\
\hline $4384.7 \mathrm{c}$ & 2.516 & 5.236 & 1.4 & 0.1455 \\
\hline $4384.7 \mathrm{~d}$ & 2.519 & 5.761 & 1.3 & 0.1521 \\
\hline $4384.6 \mathrm{c}$ & 2.523 & 5.833 & 0.9 & 0.1397 \\
\hline
\end{tabular}

Table9-2 Synthetic brine composition (g/L)

\begin{tabular}{|c|c|c|c|c|c|c|}
\hline Composition & $\begin{array}{l}\mathrm{Na} \\
\mathrm{Cl}\end{array}$ & $\begin{array}{l}\mathrm{K} \\
\mathrm{Cl}\end{array}$ & $\begin{array}{l}\mathrm{CaC} \\
\mathrm{I}_{2}\end{array}$ & $\begin{array}{l}\mathrm{Mg} \\
\mathrm{Cl}_{2}\end{array}$ & $\begin{array}{l}\mathrm{Na}_{2} \\
\mathrm{SO}_{4}\end{array}$ & TDS \\
\hline seawater & 28 & $\begin{array}{l}0.9 \\
35\end{array}$ & $\begin{array}{l}2.37 \\
9\end{array}$ & $\begin{array}{l}5.36 \\
5\end{array}$ & - & $\begin{array}{l}36.7 \\
79\end{array}$ \\
\hline $\begin{array}{l}\text { San Andres } \\
\text { Res. }\end{array}$ & $\begin{array}{l}61 . \\
77 \\
\mathbf{3}\end{array}$ & - & $\begin{array}{l}5.94 \\
4\end{array}$ & $\begin{array}{l}1.78 \\
7\end{array}$ & $\begin{array}{l}6.28 \\
0\end{array}$ & $\begin{array}{l}75.7 \\
84\end{array}$ \\
\hline
\end{tabular}

Table 9-3 Results for oil recovery for imbibition of brine followed by surfactant solution

\begin{tabular}{|l|c|c|c|c|l|c|}
\hline \multicolumn{1}{|c|}{ Cores } & $\mathrm{K}_{\mathrm{g}}, \mathrm{md}$ & $\phi, \%$ & $\begin{array}{c}\text { OR for imb. } \\
\text { of water, } \\
\% \text { OOIP }\end{array}$ & $\begin{array}{c}\mathrm{Na}_{2} \mathrm{SO}_{4}, \\
\mathrm{ppm}\end{array}$ & Surfactant & $\begin{array}{c}\Delta \text { OR for imb. of } \\
\text { surfactant, \%OOIP }\end{array}$ \\
\hline $4378.9 \mathrm{~d}$ & 144 & 0.1384 & 5.9 & 0 & $\mathrm{~T} 91-8$ & 14.0 \\
\hline $4380 \mathrm{~b}$ & 131 & 0.1108 & 5.9 & 4750 & $\mathrm{~T} 91-8$ & 16.1 \\
\hline $4380 \mathrm{a}$ & 125 & 0.1061 & 6.9 & 1500 & $\mathrm{~T} 91-8$ & 14.9 \\
\hline $4384.9 \mathrm{~d}$ & 82.4 & 0.1598 & 18.8 & 3150 & $\mathrm{~T} 91-8$ & 16.1 \\
\hline $4379.4 \mathrm{c}$ & 47.3 & 0.0810 & 4.6 & 6280 & $\mathrm{~T} 91-8$ & 0 \\
\hline & & & & & & 18.6 \\
\hline $4378.8 \mathrm{c}$ & 1.6 & 0.1384 & 6.2 & 3150 & $\mathrm{~T} 91-8$ & 1.5 \\
\hline $4384.6 \mathrm{~d}$ & 1.6 & 0.1308 & 32.5 & 4750 & $\mathrm{~T} 91-8$ & 5.3 \\
\hline $4384.7 \mathrm{c}$ & 1.4 & 0.1455 & 37.5 & 6280 & $\mathrm{~T} 91-8$ & 9.2 \\
\hline $4384.7 \mathrm{~d}$ & 1.3 & 0.1521 & 35.6 & 0 & $\mathrm{~T} 91-8$ & 6.2 \\
\hline $4384.6 \mathrm{c}$ & 0.9 & 0.1397 & 39.1 & 1500 & $\mathrm{~T} 91-8$ & \\
\hline
\end{tabular}


Fig. 9-1. Effect of T91-8 and $\mathrm{Na}_{2} \mathrm{SO}_{4}$ on enhanced oil recovery through spontaneous imbibition for San Andres dolomites (solid symbols correspond to initial imbibition of sea water)

la

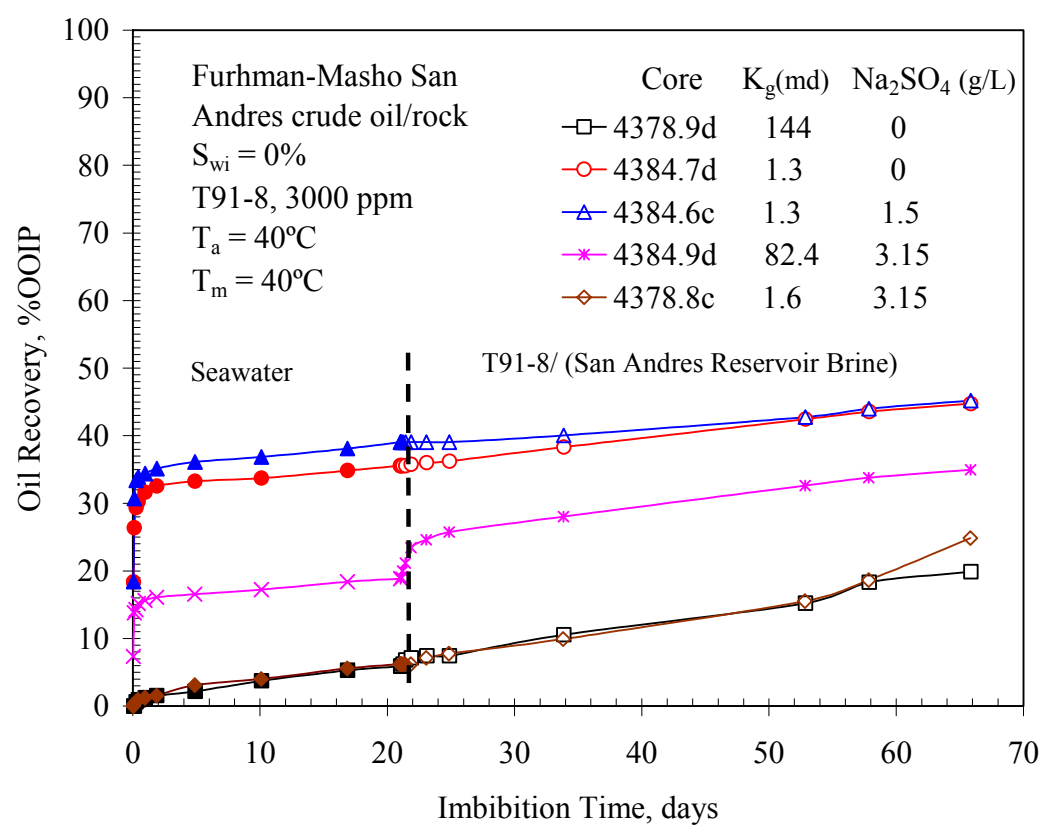

$1 b$

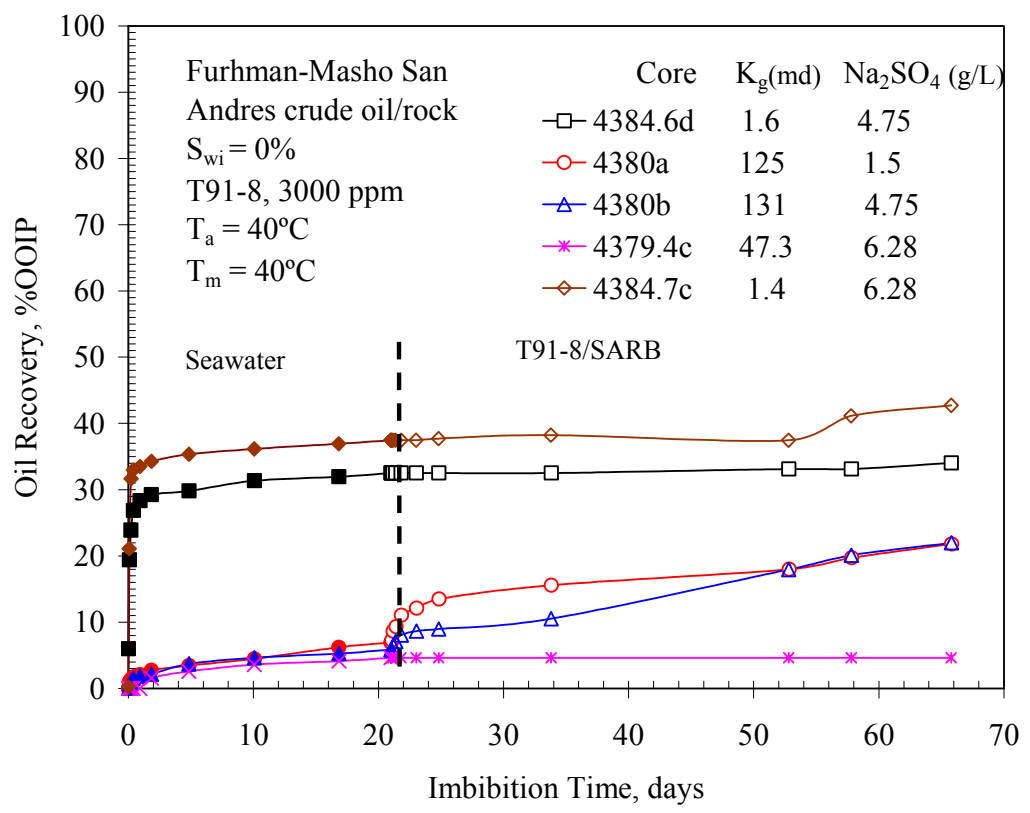




\section{CONCLUSIONS}

\section{Task 6}

1. A technique involving centrifuging and solidification of liquid resins enabled the distribution of wetting and non-wetting phases and interfacial areas to be determined from thin sections for sandstone and carbonate.

2. The total area created by invasion of the non-wetting phase increased linearly with decrease in wetting phase saturation for both sandstone and carbonate. The interfacial area between the wetting and non-wetting phase was always only a small fraction of the non-wetting phase/solid area.

3. Comparison of created surface area with rock surface area measured by gas adsorption showed that, after primary drainage to $20 \%$ wetting phase saturation, most of the rock surface area is still overlain by bulk wetting phase.

4. The efficiency of conversion of work of displacement (based on air/brine capillary pressure measurements) to surface free energy for displacement to $20 \%$ wetting phase saturation was only $36 \%$ for sandstones and $16 \%$ for limestone. These values are very low compared to the value of $85 \%$ measured for sphere packs.

5. Drainage of a spherical cavity provides explanation of how aspect ratio (pore body to pore throat size) and wettability can have a dominant effect on the efficiency of conversion of work of displacement to surface free energy.

Task 7/Task 8.

6. Monitoring the end pressure for one end open linear imbibition after transition to restricted COUCSI provides an estimate of the capillary pressure acting at the COUCSI imbibition front under dynamic conditions.

7. For liquid/gas imbibition, compressibility effects need special consideration in design of the tests on transition to restricted COUCSI. Raising the air pressure ahead of the front prevents the WP front from reaching the dead end of the core before the end pressure reaches its maximum value.

8. Frontal capillary pressures for water/oil and oil/air in the same permeability Berea sandstone of selected permeability scale very closely with interfacial tension.

\section{Task 9.}

9. Overall, low concentration of nonionic surfactant gave increased oil recovery from San Andres limestone by spontaneous imbibition for a wide range of permeability. Oil recovery from chalk with a cationic surfactant has been reported to give significant increase in recovery with sulfate concentration. However, sulfate concentration did not give a consistent trend for the crude oil/limestone surfactant combination used in the present work 